\title{
Poisson kernels of half-spaces in real hyperbolic spaces
}

\author{
Tomasz Byczkowski, Piotr Graczyk and Andrzej Stós
}

\begin{abstract}
We provide an integral formula for the Poisson kernel of halfspaces for Brownian motion in real hyperbolic space $\mathbb{H}^{n}$. This enables us to find asymptotic properties of the kernel. We also show convergence to the Poisson kernel of the whole space $\mathbb{H}^{n}$. For $n=3$, 4 or 6 we compute explicit formulas for the Poisson kernel itself.
\end{abstract}

\section{Introduction}

In recent years there is a growing interest in research of Brownian motion on hyperbolic spaces (cf. [15], [3]). More advanced theory requires a detailed knowledge of some basic potential-theoretic objects. The most fundamental turns out to be the Poisson kernel for a domain. If we know the Poisson kernel then we are able, for example, to solve the classical Dirichlet boundary problem for a domain. Another basic object in potential theory is the Green function of a domain. The "sweeping out" method allows to recover the Green function from the Poisson kernel. The Green function of a domain is a very useful tool in solving various important problems in potential theory. In particular, it enables to determine conditions under which a reasonable potential theory of the Schrödinger operator is feasible for a given domain and a particular potential ([5]). In the classical situation of the Laplacian in $\mathbb{R}^{n}$, the exact formula for the Poisson kernel (or Green function) always simplifies the arguments as well as indicates the future areas of investigation. We believe that the same situation will occur in the case of hyperbolic spaces.

The aim of this paper is to give a representation formula for the Poisson kernel of a half-space in the real hyperbolic space $\mathbb{H}^{n}$, i.e. for the probability

2000 Mathematics Subject Classification: Primary: 60J45. Secondary: 58J65.

Keywords: Hyperbolic spaces, Brownian motion, Poisson kernel. 
distribution of the hyperbolic Brownian motion stopped when leaving a halfspace, and to use it in order to prove exact asymptotics of the kernel. Note that the boundary of the considered half-space is a horocycle in $\mathbb{H}^{n}$.

The Poisson kernel of a half-space is closely related to stable laws and functionals of the Brownian motion ([1], [2], [13], [14]). Another motivation comes from the risk theory in financial mathematics ([6]). Our kernel, up to a passage from the dimension 2 to the dimension $n$, was identified in terms of its Fourier transform in [1]. It turns out, however, that it is not sufficient for most applications. In particular, a formula for the kernel itself or its asymptotical behaviour were not identified (cf. [2, p. 589]).

From the technical point of view, the main difficulty is that the inverse Fourier transform (or the Hankel transform) leads to an integral containing Bessel functions which has oscillatory character, see (2.5) below. Moreover, for integrals like (2.5), the Lebesgue bounded convergence theorem is often not applicable (for example when $|y| \rightarrow \infty$ ) and we are left with a nontrivial problem of obtaining the asymptotics of the kernel.

The paper is organized as follows. In Section 2, after some preliminaries, we provide, for convenience of the reader, proof of the formula for the Fourier transform of the Poisson kernel of a half-space in $\mathbb{H}^{n}$. We also state a first integral formula (2.5) for the Poisson kernel of a half-space, based on the inverse Fourier transform. In Section 3, in Theorem 3.2 we obtain a second integral formula for the Poisson kernel of a half-space. This is our main representation formula. It is much more suitable for further applications than (2.5). Section 3 ends with explicit integral formulas for the Poisson kernel of a half-space that arise in lower dimensions. In Section 4 we study the above mentioned asymptotics of the Poisson kernel of a half-space in $\mathbb{H}^{n}$. We use our main representation formula from Theorem 3.2 as well as the semigroup and homogeneity properties of the Poisson kernel.

\section{Preliminaries}

Consider the half-space model of the $n$-dimensional real hyperbolic space

$$
\mathbb{H}^{n}=\left\{\left(x_{1}, \ldots, x_{n-1}, x_{n}\right) \in \mathbb{R}^{n-1} \times \mathbb{R}: x_{n}>0\right\} .
$$

The Riemannian metric and the volume element are given by

$$
\begin{aligned}
& d s^{2}=\frac{d x_{1}^{2}+\cdots+d x_{n-1}^{2}+d x_{n}^{2}}{x_{n}^{2}}, \\
& d V=\frac{d x_{1} \cdots d x_{n-1} d x_{n}}{x_{n}^{n}},
\end{aligned}
$$


and the Laplace-Beltrami operator reads as

$$
\Delta=x_{n}^{2}\left(\sum_{i=1}^{n} \partial_{i}^{2}\right)-(n-2) x_{n} \partial_{n},
$$

(here $\partial_{i}=\partial / \partial x_{i}, i=1, \ldots, n$ ).

Let $\left(B_{i}(t)\right)_{i=1, \ldots, n}$ be a family of independent classical Brownian motions on $\mathbb{R}$ with the generator $\frac{d^{2}}{d x^{2}}$ (and not $\frac{1}{2} \frac{d^{2}}{d x^{2}}$ ) i.e. the variance $E^{0} B_{i}^{2}(t)=2 t$. Then the Brownian motion on $\mathbb{H}^{n}, X=\left(X_{i}\right)_{i=1, \ldots, n}$, can be described by the following system of stochastic differential equations

$$
\left\{\begin{array}{rrrr}
d X_{1}(t) & = & X_{n}(t) d B_{1}(t) \\
d X_{2}(t) & = & & X_{n}(t) d B_{2}(t) \\
& \vdots & & \\
d X_{n}(t) & = & X_{n}(t) d B_{n}(t)-(n-2) X_{n}(t) d t
\end{array}\right.
$$

By the Itô formula one verifies that the generator of the solution of this system is $\Delta$. Moreover, it can be easily verified that the solution is given by

$$
\left\{\begin{aligned}
X_{1}(t) & =X_{1}(0)+\int_{0}^{t} X_{n}(0) \exp \left(B_{n}(s)-(n-1) s\right) d B_{1}(s) \\
X_{2}(t) & =X_{2}(0)+\int_{0}^{t} X_{n}(0) \exp \left(B_{n}(s)-(n-1) s\right) d B_{2}(s) \\
& \vdots \\
X_{n}(t) & =X_{n}(0) \exp \left(B_{n}(t)-(n-1) t\right) .
\end{aligned}\right.
$$

Convention: by $c$ (or $C$ ) we always denote a general constant that depends on $n$ and other constant parameters only. The value of these constants may change in the same string of estimates.

Below we identify the Poisson kernel (the function $y \rightarrow P_{a}(x, y)$ ) in terms of its Fourier transform. Theorems 2.1 and 2.2 cover facts that are essentially known. Similar results can be found in both [1] and [2], in a slightly different setting of more general generators on the space $\mathbb{H}^{2}$. In order to make our paper self-contained and for the convenience of the reader, we include these facts here, in the present setting of $\mathbb{H}^{n}$.

Define the projection ${ }^{\sim}$ :

$$
\mathbb{R}^{n} \ni u=\left(u_{1}, \ldots, u_{n}\right) \rightarrow \tilde{u}=\left(u_{1}, \ldots, u_{n-1}\right) \in \mathbb{R}^{n-1} .
$$

In particular, $\tilde{X}(t)=\left(X_{1}(t), \ldots, X_{n-1}(t)\right)$.

Consider a half-space $D=\left\{x \in \mathbb{H}^{n}: x_{n}>a\right\}$ for some fixed $a>0$. Define

$$
\tau=\inf \{t \geqslant 0: X(t) \notin D\}=\inf \left\{t \geqslant 0: X_{n}(t)=a\right\} .
$$

By $P_{a}(x, d y), x=\left(x_{1}, x_{2}, \ldots, x_{n}\right) \in D, y=\left(y_{1}, y_{2}, \ldots, y_{n-1}, a\right) \in \partial D$ we denote the Poisson kernel of $D$, i.e. the distribution of $X(\tau)$ starting at $x$ (since $X_{n}(\tau)=a$ it is enough to consider the distribution of $\tilde{X}(\tau)$ ). 


\section{Theorem 2.1}

$$
\begin{aligned}
\mathcal{F}\left[P_{a}(x, \cdot)\right](u) & =E^{x} e^{i\left\langle u, \tilde{X}_{\tau}\right\rangle} \\
& =e^{i\langle\tilde{x}, u\rangle}\left(\frac{x_{n}}{a}\right)^{\frac{n-1}{2}} \frac{K_{\frac{n-1}{2}}\left(|u| x_{n}\right)}{K_{\frac{n-1}{2}}(|u| a)}, \quad x \in D, u \in \mathbb{R}^{n-1},
\end{aligned}
$$

where $K_{\nu}, \nu>0$, is the modified Bessel function of the third kind, called also Macdonald function.

Proof. Since $B_{i}(t)$ are independent and $\tau$ depends only on $X_{n}$ (i.e. $B_{n}$ ) we obtain for $x=\left(\tilde{x}, x_{n}\right)=\left(x_{1}, x_{2}, \ldots, x_{n}\right)$

$$
E^{x} \exp (i\langle u, \tilde{X}(\tau)\rangle)=E^{x_{n}} \prod_{j=1}^{n-1} E^{x_{j}} \exp \left(i u_{j} X_{j}(\tau)\right) .
$$

We adopt here a useful notation that $E^{x_{j}} Y$ for the expectation with respect to $j$ 'th component $B_{j}$ of our basic $(n-1)$-dimensional Brownian motion $B=\left(B_{1}, \ldots, B_{n}\right)$, starting from $x_{n}$. We compute the integral $\int_{0}^{\tau} X_{n}(s) d B_{j}(s)$ by approximation of $\mathbf{1}_{\{s<\tau\}}(s) X_{n}(s)$ by simple processes of the form $\sum_{k} f_{k} \mathbf{1}_{\left[t_{k}, t_{k+1}\right)}$, where $f_{k} \in \mathcal{F}\left(B_{n}\left(t_{k}\right)\right)$. Using independence of the increments of $B_{j}$ and the fact that the function under the integral below is independent of $B_{j}$, we get

$$
\begin{aligned}
E^{x_{j}} \exp \left(i u_{j} X_{j}(\tau)\right) & =E^{x_{j}} \exp \left(i u_{j}\left(X_{j}(0)+\int_{0}^{\tau} X_{n} d B_{j}\right)\right) \\
& =e^{i u_{j} x_{j}} \exp \left(-u_{j}^{2} \int_{0}^{\tau} X_{n}^{2}(s) d s\right)
\end{aligned}
$$

This and (2.1) imply that

$$
E^{x} \exp (i\langle u, \tilde{X}(\tau)\rangle)=e^{i\langle\tilde{x}, u\rangle} E^{x_{n}} e^{-|u|^{2} \int_{0}^{\tau} X_{n}^{2}(s) d s}=e^{i\langle\tilde{x}, u\rangle} E^{x_{n}} e_{q}(\tau),
$$

where $e_{q}(\tau)=\exp \left(\int_{0}^{\tau} q\left(X_{n}(s)\right)\right) d s$ with $q(y)=-(|u| y)^{2}$. Observe that the function $\varphi(y)=E^{y} e_{q}(\tau)$ is by definition the gauge for the Schrödinger operator $L+q$ based on the generator $L$ of $X_{n}$ and the potential $q$. By general theory (see e.g. [5, Prop. 4.13, p. 119]) it is a solution for the Schrödinger equation. Since $d X_{n}(t)=X_{n}(t) d B_{n}(t)-(n-2) X_{n}(t) d t$, by a standard argument based on the Itô formula, we get the generator of $X_{n}$

$$
L=x_{n}^{2} \frac{d^{2}}{d x_{n}^{2}}-(n-2) x_{n} \frac{d}{d x_{n}} .
$$

Consequently, $\varphi$ satisfies the following equation

$$
y^{2} \varphi^{\prime \prime}(y)-(n-2) y \varphi^{\prime}(y)-|u|^{2} y^{2} \varphi(y)=0
$$

on the positive half-line. 
Let $\varphi(y)=y^{\frac{n-1}{2}} g(y)$. Then $\varphi^{\prime}(y)=\frac{n-1}{2} y^{\frac{n-3}{2}} g(y)+y^{\frac{n-1}{2}} g^{\prime}(y), \varphi^{\prime \prime}(y)=$ $\frac{(n-1)(n-3)}{4} y^{\frac{n-5}{2}} g(y)+(n-1) y^{\frac{n-3}{2}} g^{\prime}(y)+y^{\frac{n-1}{2}} g^{\prime \prime}(y)$ and consequently (2.2) reads as

$$
y^{2} g^{\prime \prime}(y)+y g^{\prime}(y)-\left(|u|^{2} y^{2}+((n-1) / 2)^{2}\right) g(y)=0 .
$$

Substituting $|u| y=z$ and $g(y)=h(z)$ we get

$$
z^{2} h^{\prime \prime}(z)+z h^{\prime}(z)-\left(z^{2}+((n-1) / 2)^{2}\right) h(z)=0 .
$$

This is the modified Bessel equation of order $(n-1) / 2$. Taking into account the form of the general solution of (2.3) we infer that

$$
\varphi(y)=y^{\frac{n-1}{2}}\left(c_{1} I_{\frac{n-1}{2}}(|u| y)+c_{2} K_{\frac{n-1}{2}}(|u| y)\right),
$$

for an appropriate choice of $c_{1}$ and $c_{2}$, where $I_{\frac{n-1}{2}}(\cdot)$ and $K_{\frac{n-1}{2}}(\cdot)$ are the modified Bessel function of the first and third kind, respectively. Observe that by definition $\varphi(y)$ is bounded in $y$ and $\varphi(a)=1$. Since $I_{\frac{n-1}{2}}(|u| y)$ is unbounded and $K_{\frac{n-1}{2}}(|u| y)$ is bounded when $y \rightarrow \infty$, it follows that $c_{1}=0$. From the other condition we get the normalizing constant

$$
c_{2}=\frac{1}{a^{\frac{n-1}{2}} K_{\frac{n-1}{2}}(|u| a)} .
$$

This completes the proof.

Remark. As $K_{\frac{n-1}{2}}(x) \sim \sqrt{\pi}(2 x)^{-1 / 2} e^{-x}$ when $x \rightarrow \infty$, the Fourier transform of our kernel is in $L^{1}$. Thus there exists the corresponding density which we denote by $P_{a}(x, y)$.

From Theorem 2.1 it follows that nonzero $\tilde{x}$ gives rise just to a translation of $P_{a}(x, y)$ as a function of $y$. Therefore, in what follows we may and do assume $\tilde{x}=0$. Consequently, we may simplify the notation by identifying $P_{a}(x, y)=P_{a}((0, \ldots, 0, x), y), x>0$.

For $z>0$ we define $([10,8.432 .8])$

$$
m_{\frac{n}{2}-1}(z)=\int_{0}^{\infty} e^{-u} u^{\frac{n}{2}-1}(u+2 z)^{\frac{n}{2}-1} d u=d_{n} e^{z} z^{\frac{n-1}{2}} K_{\frac{n-1}{2}}(z),
$$

where $d_{n}=\pi^{-\frac{1}{2}} 2^{\frac{n+1}{2}} \Gamma\left(\frac{n}{2}\right)$. Observe that for $n \in 2 \mathbb{N}$ the function $m_{\frac{n}{2}-1}$ is just a polynomial of the degree $\frac{n}{2}-1$. In this case we regard $m_{\frac{n}{2}-1}(z)$ as defined for all complex numbers. By [10, 8.468, p. 915], for $n \in 2 \mathbb{N}$ we get

$$
m_{\frac{n}{2}-1}(z)=d_{n} \sqrt{\frac{\pi}{2}} \sum_{j=0}^{\frac{n}{2}-1} \frac{(n-2-j) ! 2^{j+\frac{n}{2}+1}}{j !\left(\frac{n}{2}-1-j\right) !} z^{j} .
$$

In particular, $m_{0}(z)=1, m_{1}(z)=2(1+z)$ and $m_{2}(z)=8\left(z^{2}+3 z+3\right)$. 
Theorem 2.2 (Poisson kernel formula) Let $a>0, x>a$ and $y \in \mathbb{R}^{n-1}$. If $|y|>0$ then

$$
P_{a}(x, y)=\left(\frac{x}{2 \pi a}\right)^{\frac{n-1}{2}}|y|^{-\frac{n-3}{2}} \int_{0}^{\infty} \frac{K_{\frac{n-1}{2}}(r x)}{K_{\frac{n-1}{2}}(r a)} J_{\frac{n-3}{2}}(r|y|) r^{\frac{n-1}{2}} d r,
$$

and when $|y|=0$ it is understood in the limiting sense, i.e.

$$
P_{a}(x, 0)=\frac{2^{2-n}}{\Gamma\left(\frac{n-1}{2}\right)}\left(\frac{x}{\pi a}\right)^{\frac{n-1}{2}} \int_{0}^{\infty} \frac{K_{\frac{n-1}{2}}(r x)}{K_{\frac{n-1}{2}}(r a)} r^{n-2} d r .
$$

Equivalently, we can write

$$
P_{a}(x, y)=(2 \pi)^{\frac{1-n}{2}}|y|^{-\frac{n-3}{2}} \int_{0}^{\infty} e^{-r(x-a)} \frac{m_{\frac{n}{2}-1}(r x)}{m_{\frac{n}{2}-1}(r a)} r^{\frac{n-1}{2}} J_{\frac{n-3}{2}}(r|y|) d r,
$$

for $|y|>0$. The special case $|y|=0$ reads as

$$
P_{a}(x, 0)=\frac{2^{2-n} \pi^{\frac{1-n}{2}}}{\Gamma\left(\frac{n-1}{2}\right)} \int_{0}^{\infty} e^{-r(x-a)} \frac{m_{\frac{n}{2}-1}(r x)}{m_{\frac{n}{2}-1}(r a)} r^{n-2} d r .
$$

Proof. Recall that if $f$ is a radial function, $f(y)=f_{o}(|y|)$, then so is $\mathcal{F} f$ and the Fourier inversion formula in $\mathbb{R}^{n-1}$ reads, up to a factor $(2 \pi)^{-(n-1)}$, as the Hankel transform of order $(n-3) / 2$ ([9, (7.38), p. 247]):

$$
f_{o}(|y|)=(2 \pi)^{-\frac{n-1}{2}} \int_{0}^{\infty}(\mathcal{F} f)_{o}(r)(r|y|)^{1-\frac{n-1}{2}} J_{\frac{n-1}{2}-1}(r|y|) r^{n-2} d r .
$$

This gives (2.5).

Now, (2.7) is immediate and the special cases $y=0$ follow from the asymptotics of the Bessel function (see e.g. $[10,8.440]$ or $[9,(5.10)$, p. 130])

$$
J_{\nu}(z) \sim \frac{1}{2^{\nu} \Gamma(1+\nu)} z^{\nu}, \quad z \rightarrow 0 .
$$

The proof is complete.

Corollary 2.3 The Poisson kernel $P_{a}(x, y)$, as a function of three variables $(a, x, y)$, is a homogeneous function of order $-n+1$ :

$$
P_{t a}(t x, t y)=t^{-n+1} P_{a}(x, y), \quad t>0 .
$$

Proof. The homogeneity property can be derived easily from the scaling properties of the process $\left(X_{1}(t), \ldots, X_{n}(t)\right)$. This is also obvious by change of variables $\tilde{r}=t r$ in the formula (2.5) written for $P_{t a}(t x, t y)$.

Remark. Certainly, when $n \in 2 \mathbb{N}$ then also $J_{\frac{n-3}{2}}(r|y|)$ simplifies to an elementary function. This fact, however, is not very useful in what follows and we will not pursue this further. 


\section{Poisson kernel of half-space}

In this section we give a representation formula for the Poisson kernel. For $n=2$ the resulting formula coincides with the one of $\mathbb{R}^{2}$, so that below we shall always tacitly assume $n \geq 3$ (note, however, that a great part of our argument remains valid also for $n=2$ ).

From now on we use the following notation, partially introduced in the preceding section: $\lambda=x-a$.

The following technical lemma is essential in what follows.

Lemma 3.1 Let

$$
Q(z)=z-n(n-2) \frac{\lambda}{8 a x}, \quad z \in \mathbb{C} .
$$

Define $F_{\lambda}(z)$ by the following formula:

$$
\lambda F_{\lambda}(z)=\frac{(z / a) e^{\lambda z / a}(x / a)^{\frac{n-1}{2}} K_{\frac{n-1}{2}}(x z / a)-(x / a)^{\frac{n}{2}-1} Q(z / a) K_{\frac{n-1}{2}}(z)}{K_{\frac{n-1}{2}}(z)}
$$

Then

$$
F_{\lambda}(z)=O\left(z^{-1}\right), \quad z \rightarrow \infty
$$

Proof. Using the asymptotic expansions for the modified Bessel function $K_{\frac{n-1}{2}}(z)([10,8.451 .6$, p. 910$])$ we get

$$
e^{z} z^{\frac{n-1}{2}} K_{\frac{n-1}{2}}(z)=z^{\frac{n}{2}-1}\left(c_{0}+\frac{c_{1}}{2 z}+R_{2}\right)
$$

where

$$
c_{k}=c_{k}^{(n)}=\sqrt{\frac{\pi}{2}} \frac{\Gamma(n / 2+k)}{k ! \Gamma(n / 2-k)}, \quad k=0,1,
$$

$R_{2}=O\left(z^{-2}\right)$ and $|z|$ is large enough. Hence, it is enough to show

$(z / a) e^{x z / a}(x z / a)^{\frac{n-1}{2}} K_{\frac{n-1}{2}}(x z / a)-(x / a)^{\frac{n}{2}-1} Q(z / a) e^{z} z^{\frac{n-1}{2}} K_{\frac{n-1}{2}}(z)$

$$
=O\left(z^{\frac{n}{2}-2}\right), \quad z \rightarrow \infty .
$$

From (3.3) it follows that on one hand we have

$$
\begin{aligned}
(z / a) e^{x z / a} & (x z / a)^{\frac{n-1}{2}} K_{\frac{n-1}{2}}(x z / a) \\
& =c_{0}(x / a)^{\frac{n}{2}-1}\left(z^{\frac{n}{2}} / a\right)+\left(c_{1} /(2 x)\right)(x / a)^{\frac{n}{2}-1} z^{\frac{n}{2}-1}+O\left(z^{\frac{n}{2}-2}\right) .
\end{aligned}
$$


On the other hand, using $\frac{n(n-2)}{4} c_{0}=c_{1}$, we get

$$
\begin{array}{rl}
\left(\frac{x}{a}\right)^{\frac{n}{2}-1} & Q\left(\frac{z}{a}\right) e^{z} z^{\frac{n-1}{2}} K_{\frac{n-1}{2}}(z) \\
& =\left(\frac{x}{a}\right)^{\frac{n}{2}-1}\left(\frac{z}{a}-\frac{n(n-2)}{8} \frac{\lambda}{a x}\right)\left(c_{0} z^{\frac{n}{2}-1}+\frac{1}{2} c_{1} z^{\frac{n}{2}-2}+O\left(z^{\frac{n}{2}-3}\right)\right) \\
& =\frac{c_{o}}{a}\left(\frac{x}{a}\right)^{\frac{n}{2}-1} z^{\frac{n}{2}}+\left(\frac{x}{a}\right)^{\frac{n}{2}-1}\left(\frac{c_{1}}{2 a}-\frac{n(n-2)}{4} c_{0} \frac{\lambda}{2 a x}\right) z^{\frac{n}{2}-1}+O\left(z^{\frac{n}{2}-2}\right) \\
& =\frac{c_{o}}{a}\left(\frac{x}{a}\right)^{\frac{n}{2}-1} z^{\frac{n}{2}}+\left(\frac{x}{a}\right)^{\frac{n}{2}-1} \frac{c_{1}}{2 x} z^{\frac{n}{2}-1}+O\left(z^{\frac{n}{2}-2}\right) .
\end{array}
$$

Then (3.4) is obviously satisfied and the assertion follows.

Remark. The advantage of this lemma is due to the fact that we may and do use it for $z \in \mathbb{C}$. This fact is exploited below.

Observe that the function $K_{\frac{n}{2}-1}(z)$ has no zeros in $\{\Re(z) \geq 0\}$ (cf. [8, p. 62]) and hence $F_{\lambda}(z)$ is analytic in this half-plane. Moreover, by the inverse Laplace transform theorem ([9, Theorem 8.5]) together with (3.2) we get that $F_{\lambda}$ is the Laplace transform of some function $w_{\lambda}$, i.e.

$$
F_{\lambda}(z)=\int_{0}^{\infty} e^{-z v} w_{\lambda}(v) d v, \quad \Re(z)>0,
$$

under the additional condition that for some $b>0$ the following limit

$$
\lim _{r \rightarrow \infty} \frac{1}{2 \pi i} \int_{b-i r}^{b+i r} F_{\lambda}(z) e^{v z} d z
$$

exists for all $v>0$ and it is a piecewise continuous function of $v$ admitting the Laplace transform. Then the limit is equal to $w_{\lambda}(v)$.

The existence of the above limit is shown in Theorem 3.3, together with an explicit formula for the function $w_{\lambda}$ itself.

We are ready to state our representation formula. Recall that $\lambda=x-a$.

\section{Theorem 3.2}

$$
P_{a}(x, y)=\frac{\Gamma\left(\frac{n}{2}-1\right)}{2 \pi^{n / 2}} \frac{\lambda}{\left(\lambda^{2}+|y|^{2}\right)^{n / 2}} \int_{0}^{\infty} \frac{w_{\lambda}(v) L(\lambda, y, v)}{\left((\lambda+a v)^{2}+|y|^{2}\right)^{\frac{n}{2}-1}} d v,
$$

with $L(\lambda, y, v)$ defined by

$$
\frac{L(\lambda, y, v)}{\left(\lambda^{2}+|y|^{2}\right)\left((\lambda+a v)^{2}+|y|^{2}\right)^{\frac{n}{2}-1}}
$$

$$
=\left(\frac{\lambda^{2}+|y|^{2}}{(\lambda+a v)^{2}+|y|^{2}}\right)^{\frac{n}{2}-1}-1+\frac{\left(\frac{n}{2}-1\right) a v(2 \lambda+a v)}{\lambda^{2}+|y|^{2}} .
$$


Proof. Recall that for $\nu>0$ we have ([7, vol I, (7) and (8) p. 182] or [10, 17.13.43, 17.13.44])

$$
\int_{0}^{\infty} e^{-\lambda r} r^{\nu} J_{\nu-1}(r|y|) d r=2^{\nu} \pi^{-\frac{1}{2}} \Gamma\left(\nu+\frac{1}{2}\right)|y|^{\nu-1} \frac{\lambda}{\left(\lambda^{2}+|y|^{2}\right)^{\nu+\frac{1}{2}}}
$$

and

$$
\int_{0}^{\infty} e^{-\lambda r} r^{\nu-1} J_{\nu-1}(r|y|) d r=2^{\nu-1} \pi^{-\frac{1}{2}} \Gamma\left(\nu-\frac{1}{2}\right)|y|^{\nu-1} \frac{1}{\left(\lambda^{2}+|y|^{2}\right)^{\nu-\frac{1}{2}}} .
$$

For $z=r a$ we have

$$
\lambda F_{\lambda}(r a)=\frac{r m_{\frac{n}{2}-1}(r x)-(x / a)^{\frac{n}{2}-1} Q(r) m_{\frac{n}{2}-1}(r a)}{m_{\frac{n}{2}-1}(r a)},
$$

SO

$$
\frac{r m_{\frac{n}{2}-1}(r x)}{m_{\frac{n}{2}-1}(r a)}=\left(\frac{x}{a}\right)^{\frac{n}{2}-1} Q(r)+\lambda F_{\lambda}(r a)
$$

and hence by $(2.4)$

$$
\left(\frac{x}{a}\right)^{\frac{n-1}{2}} \frac{r K_{\frac{n-1}{2}}(r x)}{K_{\frac{n-1}{2}}(r a)}=e^{-\lambda r}\left[\left(\frac{x}{a}\right)^{\frac{n}{2}-1} Q(r)+\lambda F_{\lambda}(r a)\right] .
$$

Putting this into the Hankel transform formula (2.5) and using (3.5) we get

$$
\begin{gathered}
\frac{2 \pi^{n / 2}}{\Gamma\left(\frac{n}{2}-1\right)} P_{a}(x, y)=\frac{\lambda(n-2)}{2}\left(\frac{x}{a}\right)^{\frac{n}{2}-1}\left[\frac{2}{\left(\lambda^{2}+|y|^{2}\right)^{\frac{n}{2}}}-\frac{1}{4 x a} \frac{n}{\left(\lambda^{2}+|y|^{2}\right)^{\frac{n}{2}-1}}\right] \\
+\lambda \int_{0}^{\infty} \frac{w_{\lambda}(v)}{\left((\lambda+a v)^{2}+|y|^{2}\right)^{\frac{n}{2}-1}} d v .
\end{gathered}
$$

Putting $r=0$ in (3.9) we get

$$
\begin{aligned}
\lambda F_{\lambda}(0) & =\lambda \int_{0}^{\infty} w_{\lambda}(v) d v \\
& =-(x / a)^{\frac{n}{2}-1} Q(0)=n(n-2)(x / a)^{\frac{n}{2}-1} \frac{\lambda}{8 x a}
\end{aligned}
$$

so that

$$
\lambda\left(F_{\lambda}(r a)-F_{\lambda}(0)\right) m_{\frac{n}{2}-1}(r a)=r m_{\frac{n}{2}-1}(r x)-(x / a)^{\frac{n}{2}-1} r m_{\frac{n}{2}-1}(r a) .
$$

Dividing both sides by $r$ and taking the limit $r \rightarrow 0$ we obtain

$$
\lambda a F_{\lambda}^{\prime}(0)=-\lambda a \int_{0}^{\infty} v w_{\lambda}(v) d v=1-(x / a)^{\frac{n}{2}-1} .
$$


We used the fact that $v w_{\lambda}(v)$ allows the Laplace transform which is evident from Theorem 3.3. Hence

$$
(x / a)^{\frac{n}{2}-1}=1+\lambda a \int_{0}^{\infty} v w_{\lambda}(v) d v .
$$

Moreover,

$$
\lambda\left[\left(F_{\lambda}(r a)-F_{\lambda}(0)\right)-r a F_{\lambda}^{\prime}(0)\right] m_{\frac{n}{2}-1}(r a)=r m_{\frac{n}{2}-1}(r x)-r m_{\frac{n}{2}-1}(r a) .
$$

Again, dividing both sides by $(r a)^{2}$, letting $r \rightarrow 0$ and using $m_{\frac{n}{2}-1}(0)=$ $m_{\frac{n}{2}-1}^{\prime}(0)$ we get

$$
\lambda F_{\lambda}^{\prime \prime}(0) / 2=\lambda / a^{2}
$$

so that

$$
1=\frac{a^{2}}{2} \int_{0}^{\infty} v^{2} w_{\lambda}(v) d v
$$

The facts that $F_{\lambda}^{\prime \prime}(0)$ exists and that the function $v^{2} w_{\lambda}(v)$ admits the Laplace transform follow from Theorem 3.3. Consequently, we have

$$
\begin{aligned}
\left(\frac{x}{a}\right)^{\frac{n}{2}-1} & =1+\lambda a \int_{0}^{\infty} v w_{\lambda}(v) d v \\
& =\frac{a^{2}}{2} \int_{0}^{\infty} v^{2} w_{\lambda}(v) d v+\lambda a \int_{0}^{\infty} v w_{\lambda}(v) d v \\
& =\frac{1}{2} \int_{0}^{\infty} a v(2 \lambda+a v) w_{\lambda}(v) d v
\end{aligned}
$$

Finally, $2 \pi^{n / 2} \Gamma\left(\frac{n}{2}-1\right)^{-1} P_{a}(x, y)$ is equal to

$$
\begin{gathered}
\frac{\lambda(n-2)}{2} \int_{0}^{\infty} \frac{a v(2 \lambda+a v) w_{\lambda}(v)}{\left(\lambda^{2}+|y|^{2}\right)^{\frac{n}{2}}} d v-\lambda \int_{0}^{\infty} \frac{w_{\lambda}(v)}{\left(\lambda^{2}+|y|^{2}\right)^{\frac{n}{2}-1}} d v \\
+\lambda \int_{0}^{\infty} \frac{w_{\lambda}(v)}{\left((\lambda+a v)^{2}+|y|^{2}\right)^{\frac{n}{2}-1}} d v \\
=\frac{\lambda}{\left(\lambda^{2}+|y|^{2}\right)^{\frac{n}{2}}} \int_{0}^{\infty} \frac{w_{\lambda}(v) L(\lambda, y, v)}{\left((\lambda+a v)^{2}+|y|^{2}\right)^{\frac{n}{2}-1}} d v
\end{gathered}
$$

and the assertion follows.

Below we give a description of the function $w_{\lambda}$. The formula depends on the zeros of the function $K_{\frac{n-1}{2}}(z)$. Even if in general the values of these zeros are not given explicitly, we are able to prove some important properties (as boundedeness or asymptotics) of $w_{\lambda}$, which are essential in applications. Moreover, in lower dimensions we provide explicit formulas as well (see Section 3). 
The function $K_{\frac{n-1}{2}}(z)$ extends to an entire function when $n$ is even and has a holomorphic extension to $\mathbb{C} \backslash(-\infty, 0]$ when $n$ is odd. Denote the set of zeros of the function $K_{\frac{n-1}{2}}(z)$ by $Z=\left\{z_{1}, \ldots, z_{k_{n}}\right\}$. We now provide some information about these zeros, needed in the sequel (cf. [8, p.62]). Recall that in the case of even dimensions the functions $m_{\frac{n}{2}-1}(z)$ are polynomials of degree $\frac{n}{2}-1$. They always have the same zeros as $K_{\frac{n-1}{2}}$, so $k_{n}=(n / 2)-1$ when $n \in 2 \mathbb{N}$. For $n=2 k+1, k_{n}$ is the even number closest to $(n / 2)-1$. In particular, we have $k_{3}=0$, and for $n=5$ and 7 we have $k_{n}=2$. The functions $K_{\frac{n-1}{2}}$ and $K_{\frac{n-3}{2}}$ have no common zeros.

In order to describe the function $w_{\lambda}$ we introduce additional notation. Let us define

$$
w_{1, \lambda}(v)=-\frac{(x / a)^{\frac{n-1}{2}}}{\lambda a} \sum_{i=1}^{k_{n}} \frac{z_{i} e^{\lambda z_{i} / a} K_{\frac{n-1}{2}}\left(x z_{i} / a\right)}{K_{\frac{n-3}{2}}\left(z_{i}\right)} e^{z_{i} v},
$$

and $w_{1}^{*}(v)=\sup _{0<\lambda \leqslant a}\left|w_{1, \lambda}(v)\right|$.

Using the functions $m_{\frac{n}{2}-1}$, the formula (3.13) reads as follows:

$$
w_{1, \lambda}(v)=\frac{-1}{(n-2) \lambda a} \sum_{i=1}^{k_{n}} \frac{m_{\frac{n}{2}-1}\left(x z_{i} / a\right)}{m_{\frac{n}{2}-2}\left(z_{i}\right)} e^{z_{i} v} .
$$

We define additionally in the case of $n$ odd

$$
\begin{aligned}
& w_{2, \lambda}(v)=(-1)^{\frac{n+1}{2}} \frac{(x / a)^{\frac{n-1}{2}}}{\lambda a} \\
& \times \int_{0}^{\infty} \frac{I_{\frac{n-1}{2}}(x u / a) K_{\frac{n-1}{2}}(u)-I_{\frac{n-1}{2}}(u) K_{\frac{n-1}{2}}(x u / a)}{K_{\frac{n-1}{2}}^{2}(u)+\pi^{2} I_{\frac{n-1}{2}}^{2}(u)} e^{-\lambda u / a} e^{-v u} u d u
\end{aligned}
$$

and, as before $w_{2}^{*}(v)=\sup _{0<\lambda \leqslant a}\left|w_{2, \lambda}(v)\right|$.

We also need the following asymptotic formulas for the modified Bessel functions $I_{\nu}, K_{\nu}$ : For $u \geqslant 1$ we have ([9], [10])

$$
I_{\nu}(u)=(2 \pi u)^{-\frac{1}{2}} e^{u}\left[1+E_{1}(u)\right], \quad K_{\nu}(u)=\pi^{\frac{1}{2}}(2 u)^{-\frac{1}{2}} e^{-u}\left[1+E_{2}(u)\right],
$$

where $E_{1}(u), E_{2}(u)=O\left(u^{-1}\right), u \rightarrow \infty$.

When $u \rightarrow 0$ we have for $\nu>0$ :

$$
I_{\nu}(u) \sim c_{\nu} u^{\nu}, \quad K_{\nu}(u) \sim c_{\nu}^{\prime} u^{-\nu},
$$

with $c_{\nu}=2^{-\nu} / \Gamma(\nu+1)$ and $c_{\nu}^{\prime}=2^{\nu-1} \Gamma(\nu)$. Whenever $\nu=0$ one has $I_{0}(u) \sim 1, K_{0}(u) \sim \log (2 / u)$. We now formulate and prove our representation theorem for the function $w_{\lambda}$. 
96 T. Byczkowski, P. Graczyk and A. Stós

Theorem 3.3 In the even dimensions

$$
w_{\lambda}(v)=w_{1, \lambda}(v)
$$

while, in the odd dimensions

$$
w_{\lambda}(v)=w_{1, \lambda}(v)+w_{2, \lambda}(v) .
$$

Moreover, we have $\left|w_{i}^{*}(v)\right| \leqslant C_{i}(n, a), i=1,2$ and

$$
\begin{gathered}
w_{1}(v)=\lim _{x \rightarrow a+} w_{1, \lambda}(v)=\frac{1}{a^{2}} \sum_{i=1}^{k_{n}} z_{i}^{2} e^{z_{i} v} ; \\
(-1)^{\frac{n+1}{2}} w_{2, \lambda}(v) \geqslant 0, \quad v \geqslant 0, \quad(n \text { odd }) ; \\
w_{2}(v)=\lim _{x \rightarrow a+} w_{2, \lambda}(v)=\frac{(-1)^{\frac{n+1}{2}}}{a^{2}} \int_{0}^{\infty} \frac{u e^{-v u} d u}{K_{\frac{n-1}{2}}^{2}(u)+\pi^{2} I_{\frac{n-1}{2}}^{2}(u)}, \quad(n \text { odd }) ; \\
\int_{0}^{\infty} v^{k} w_{1}^{*}(v) d v<\infty, \quad k=1,2, \ldots ; \quad \int_{0}^{\infty} v^{n-1} w_{2}^{*}(v) d v<\infty ; \\
\lim _{v \rightarrow \infty} v^{k} w_{1, \lambda}(v)=0, \quad k=1,2, \ldots ; \\
\lim _{v \rightarrow \infty} v^{n+1} w_{2, \lambda}(v)=\frac{(-1)^{\frac{n+1}{2}} n !}{2^{n-2} \Gamma\left(\frac{n-1}{2}\right) \Gamma\left(\frac{n+1}{2}\right)} \frac{(x / a)^{n-1}-1}{\lambda a}, \quad(n \text { odd }) .
\end{gathered}
$$

Proof. We recall the basic formula (3.1)

$$
\lambda F_{\lambda}(z)=\frac{(z / a) e^{\lambda z / a}(x / a)^{\frac{n-1}{2}} K_{\frac{n-1}{2}}(x z / a)-(x / a)^{\frac{n}{2}-1} Q(z / a) K_{\frac{n-1}{2}}(z)}{K_{\frac{n-1}{2}}(z)} .
$$

By standard rules for computing residues of meromorphic functions and using the following formula for derivatives of Bessel functions

$$
\frac{d}{d z}\left(z^{\nu} K_{\nu}(z)\right)=-z^{\nu} K_{\nu-1}(z)
$$

(cf. [8, 7.11(22), p. 79]) we obtain

$$
\operatorname{Res}_{z_{i}} F_{\lambda}=-\frac{(x / a)^{\frac{n-1}{2}}}{\lambda a} \frac{z_{i} e^{\lambda z_{i} / a} K_{\frac{n-1}{2}}\left(x z_{i} / a\right)}{K_{\frac{n-3}{2}}\left(z_{i}\right)} .
$$

Using the functions $m_{\frac{n}{2}-1}$, we obtain

$$
\operatorname{Res}_{z_{i}} F_{\lambda}=\frac{-1}{(n-2) \lambda a} \frac{m_{\frac{n}{2}-1}\left(x z_{i} / a\right)}{m_{\frac{n}{2}-2}\left(z_{i}\right)} .
$$


As mentioned before (see (3.5) and (3.6)), by the inversion theorem for the Laplace transform we have

$$
w_{\lambda}(v)=\frac{1}{2 \pi i} \lim _{r \rightarrow \infty} \int_{b-i r}^{b+i r} F_{\lambda}(z) e^{z v} d z
$$

for some $b>0$. We show the existence of the above limit together with computing formula for the function $w_{\lambda}$.

The technique of integration is different in even and odd dimensions. This is due to the fact that in the first case the function under the integral extends to a meromorphic one while in the odd dimension we have to deal with a branch cut.

For $n \in 2 \mathbb{N}$ we choose any $b>0$. All the zeros of $m_{\frac{n}{2}-1}(z)$ satisfy $\Re\left(z_{i}\right)<b$ (actually, we have in general $\Re\left(z_{i}\right)<0, i=1, \ldots, s$, cf. [8, p. 62]). To calculate $w_{\lambda}$ we integrate over the rectangular contour with corners at $b-i r, b+i r,-r-i r,-r+i r$. By (3.2) we infer that integrals over the upper, left, and bottom side of the rectangle tend to 0 as $r \rightarrow \infty$. Hence, by the residue theorem, the limit in (3.6) exists and is equal to the sum of all residues of the function $F_{\lambda}(z) e^{z \lambda}$. Thus, we have $w_{\lambda}=w_{1, \lambda}$ and the assertion follows.

In the odd dimensions, however, the function under integral is no longer meromorphic. We make the branch cut along the negative real axis $(-\infty, 0]$ and change the contour of integration to wrap around this line (see the picture).

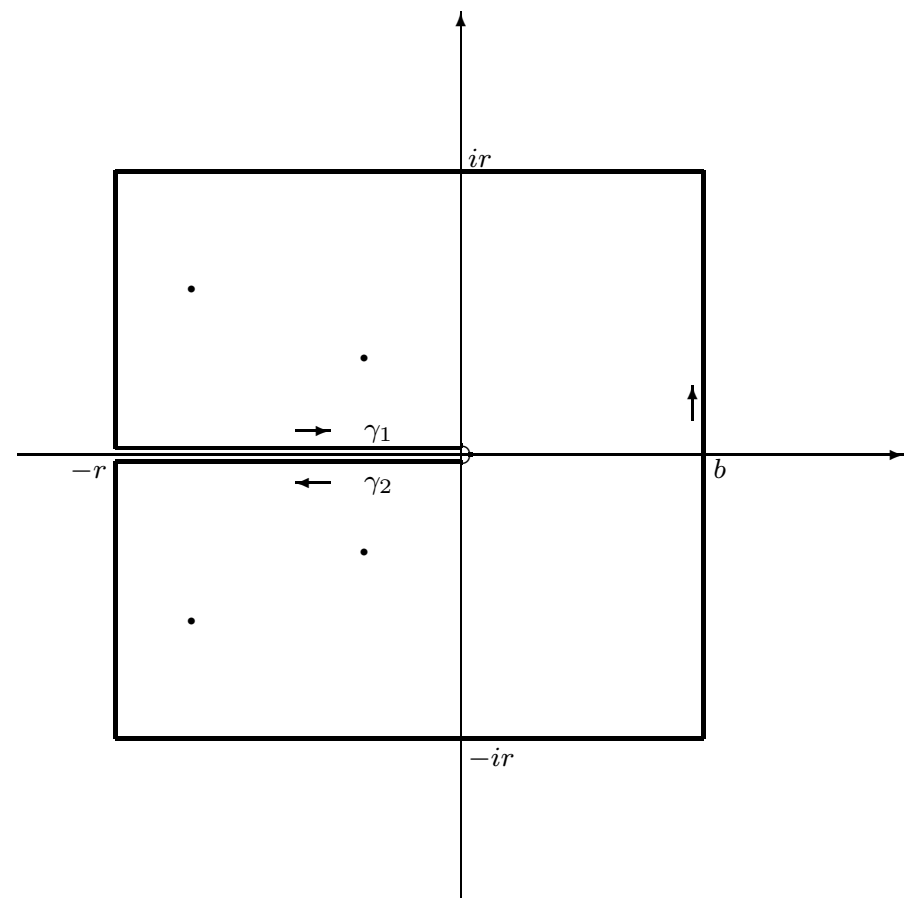


First, we examine behaviour of our function near the negative axis $(-\infty, 0)$. For $z=-y(y>0)$ we have (see $[8,(45)$, p. 80])

$$
\begin{aligned}
& \lim _{\epsilon \rightarrow 0+} K_{\frac{n-1}{2}}(-y+i \epsilon)=e^{\frac{i \pi(1-n)}{2}} K_{\frac{n-1}{2}}(y)-i \pi I_{\frac{n-1}{2}}(y), \\
& \lim _{\epsilon \rightarrow 0+} K_{\frac{n-1}{2}}(-y-i \epsilon)=e^{\frac{i \pi(n-1)}{2}} K_{\frac{n-1}{2}}(y)+i \pi I_{\frac{n-1}{2}}(y) .
\end{aligned}
$$

Now, observe that, similarly as before, the integrals over the left, upper and bottom side of our rectangular contour vanish as $r \rightarrow \infty$ by (3.2). The same holds true for the half-circle with radius $\epsilon \rightarrow 0$ around the origin. Note that the branch cut and the residues for $F_{\lambda}(z)$ are due to the term

$$
\tilde{F}_{\lambda}(z)=\frac{z(x / a)^{\frac{n-1}{2}} e^{\lambda z / a} K_{\frac{n-1}{2}}(x z / a)}{\lambda a K_{\frac{n-1}{2}}(z)}
$$

the rest of the function $F_{\lambda}(z)$ being holomorphic in $\mathbb{C}$. Therefore

$$
\frac{1}{2 \pi i} \int_{b-i r}^{b+i r} F_{\lambda}(z) e^{z v} d z=w_{1, \lambda}(v)-\frac{1}{2 \pi i}\left(\int_{\gamma_{1}}+\int_{\gamma_{2}}\right) \tilde{F}_{\lambda}(z) e^{z v} d z .
$$

After taking the limits $r \rightarrow \infty$ and $\epsilon \rightarrow 0$, we get

$$
\begin{aligned}
& \left(\int_{\gamma_{1}}+\int_{\gamma_{2}}\right) \tilde{F}_{\lambda}(z) e^{z v} d z \\
& =\frac{(x / a)^{\frac{n-1}{2}}}{\lambda a}\left(-\int_{0}^{\infty} \frac{u\left(e^{\frac{i \pi(1-n)}{2}} K_{\frac{n-1}{2}}(x u / a)-i \pi I_{\frac{n-1}{2}}(x u / a)\right)}{e^{\frac{i \pi(1-n)}{2}} K_{\frac{n-1}{2}}(u)-i \pi I_{\frac{n-1}{2}}(u)} e^{-\lambda u / a} e^{-v u} d u\right. \\
& \left.\quad+\int_{0}^{\infty} \frac{u\left(e^{\frac{i \pi(n-1)}{2}} K_{\frac{n-1}{2}}(x u / a)+i \pi I_{\frac{n-1}{2}}(x u / a)\right)}{e^{\frac{i \pi(n-1)}{2}} K_{\frac{n-1}{2}}(u)+i \pi I_{\frac{n-1}{2}}(u)} e^{-\lambda u / a} e^{-v u} d u\right) \\
& =(-1)^{\frac{n+1}{2}} \frac{(x / a)^{\frac{n-1}{2}}}{\lambda a} 2 \pi i \\
& \quad \times \int_{0}^{\infty} \frac{\left.u\left[I_{\frac{n-1}{2}}(u) K_{\frac{n-1}{2}}(x u / a)-I_{\frac{n-1}{2}}(x u / a) K_{\frac{n-1}{2}} u\right)\right]}{K_{\frac{n-1}{2}}^{2}(u)+\pi^{2} I_{\frac{n-1}{2}}^{2}(u)} e^{-\lambda u / a} e^{-v u} d u .
\end{aligned}
$$

This ends the proof of the first part of the theorem.

All what remains is to show the corresponding properties of the functions $w_{i, \lambda}, i=1,2$. We begin with $w_{1, \lambda}$, which is easier to analyze. First of all, observe that $\Re\left(z_{i}\right)<0$, so for fixed $\lambda>0$ the function $w_{1, \lambda}$ is bounded 
and $\lim _{v \rightarrow \infty} v^{k} w_{1, \lambda}(v)=0$, for all $k=1,2, \ldots$ To see what happens when $\lambda \rightarrow 0$ we use the formula for the residue of $F_{\lambda}$ (see (3.17)), together with the Lagrange formula. Since $K_{\frac{n-1}{2}}\left(z_{i}\right)=0$ we get

$$
\begin{aligned}
\operatorname{Res}_{z_{i}} F_{\lambda} & =\frac{1}{\lambda} \frac{\left(z_{i} / a\right) e^{\lambda z_{i} / a}\left(x z_{i} / a\right)^{\frac{n-1}{2}} K_{\frac{n-1}{2}}\left(x z_{i} / a\right)}{-z_{i}^{\frac{n-1}{2}} K_{\frac{n-3}{2}}\left(z_{i}\right)} \\
& =\frac{-e^{\lambda z_{i} / a}}{a^{2}} \frac{z_{i}}{z_{i}^{\frac{n-3}{2}} K_{\frac{n-3}{2}}\left(z_{i}\right)} \frac{\left(x z_{i} / a\right)^{\frac{n-1}{2}} K_{\frac{n-1}{2}}\left(x z_{i} / a\right)-z_{i}^{\frac{n-1}{2}} K_{\frac{n-1}{2}}\left(z_{i}\right)}{\left(x z_{i} / a\right)-z_{i}} \\
& =\frac{e^{\lambda z_{i} / a}}{a^{2}} \frac{z_{i}}{z_{i}^{\frac{n-3}{2}} K_{\frac{n-3}{2}}\left(z_{i}\right)}\left(\xi z_{i}\right)^{\frac{n-1}{2}} K_{\frac{n-3}{2}}\left(\xi z_{i}\right) \\
& \rightarrow \frac{z_{i} z_{i}^{\frac{n-1}{2}} K_{\frac{n-3}{2}}\left(z_{i}\right)}{a^{2} z_{i}^{\frac{n-3}{2}} K_{\frac{n-3}{2}}\left(z_{i}\right)}=\left(\frac{z_{i}}{a}\right)^{2},
\end{aligned}
$$

because $1<\xi<x / a$ and $\xi \rightarrow 1$ as $\lambda \rightarrow 0$.

Furthermore, for $0<\lambda \leqslant a$ we have

$$
\left|\operatorname{Res}_{z_{i}} F_{\lambda}\right| \leqslant\left(\frac{\left|z_{i}\right|}{a}\right)^{2} 2^{\frac{n-1}{2}} \sup _{1 \leqslant \xi \leqslant 2}\left|\frac{K_{\frac{n-3}{2}}\left(\xi z_{i}\right)}{K_{\frac{n-3}{2}}\left(z_{i}\right)}\right| .
$$

Since $\Re\left(z_{i}\right)<0$, we have obtained that $\left|w_{1}^{*}(v)\right|$ is bounded by a constant $C_{1}(n, a)$ and that $w_{1}^{*}$ integrates all powers of $v$.

We now prove the corresponding statements for $w_{2, \lambda}$. Observe that the numerator in (3.14) is equal to

$$
K_{\frac{n-1}{2}}(u) K_{\frac{n-1}{2}}(x u / a)\left(\frac{I_{\frac{n-1}{2}}(x u / a)}{K_{\frac{n-1}{2}}(x u / a)}-\frac{I_{\frac{n-1}{2}}(u)}{K_{\frac{n-1}{2}}(u)}\right)
$$

and hence is positive, because the function $I_{\nu}(u) / K_{\nu}(u), u>0$, is obviously increasing.

Using the Lagrange formula once again and taking into account

$$
\left(z^{\nu} I_{\nu}(z)\right)^{\prime}=z^{\nu} I_{\nu-1}(z)
$$

(see $[8,7.11(19)$, p. 79$])$ we obtain for $0<\lambda \leqslant a$ 


$$
\begin{aligned}
\frac{1}{\lambda} \frac{\left[I_{\frac{n-1}{2}}(x u / a) K_{\frac{n-1}{2}}(u)-I_{\frac{n-1}{2}}(u) K_{\frac{n-1}{2}}(x u / a)\right]}{K_{\frac{n-1}{2}}^{2}(u)+\pi^{2} I_{\frac{n-1}{2}}^{2}(u)} \\
=\frac{\lambda u}{a} \frac{1}{\lambda} \frac{\left[(x u / a)^{\frac{n-1}{2}} I_{\frac{n-1}{2}}(x u / a)-u^{\frac{n-1}{2}} I_{\frac{n-1}{2}}(u)\right]}{(x u / a)-u} \\
\times \frac{K_{\frac{n-1}{2}}(u)}{(x u / a)^{\frac{n-1}{2}}\left[K_{\frac{n-1}{2}}^{2}(u)+\pi^{2} I_{\frac{n-1}{2}}^{2}(u)\right]} \\
\quad \times \frac{(x u / a)-u}{a} \frac{1}{\lambda} \frac{\left((x u / a)^{\frac{n-1}{2}} K_{\frac{n-1}{2}}(x u / a)-u^{\frac{n-1}{2}} K_{\frac{n-1}{2}}(u)\right]}{(x u / a)^{\frac{n-1}{2}}\left[K_{\frac{n-1}{2}}^{2}(u)+\pi^{2} I_{\frac{n-1}{2}}^{2}(u)\right]} \\
=\frac{u}{a} \frac{\left(\xi_{1} u\right)^{\frac{n-1}{2}} I_{\frac{n-3}{2}}\left(\xi_{1} u\right) K_{\frac{n-1}{2}}(u)+\left(\xi_{2} u\right)^{\frac{n-1}{2}} I_{\frac{n-1}{2}}(u) K_{\frac{n-3}{2}}\left(\xi_{2} u\right)}{(x u / a)^{\frac{n-1}{2}}\left[K_{\frac{n-1}{2}}^{2}(u)+\pi^{2} I_{\frac{n-1}{2}}^{2}(u)\right]} \\
\rightarrow \frac{u}{a} \frac{\left[I_{\frac{n-3}{2}}(u) K_{\frac{n-1}{2}}(u)+I_{\frac{n-1}{2}}(u) K_{\frac{n-3}{2}}(u)\right]}{K_{\frac{n-1}{2}}^{2}(u)+\pi^{2} I_{\frac{n-1}{2}}^{2}(u)} \\
\frac{1}{a} \frac{K_{\frac{n-1}{2}}^{2}(u)+\pi^{2} I_{\frac{n-1}{2}}^{2}(u)}{}
\end{aligned}
$$

where for the last equality we used $[8,7.11(39)$, p. 80]. Here the convergence takes place when $\lambda \rightarrow 0$ and $0<\xi_{1}, \xi_{2} \leqslant x / a \leqslant 2$ and $\xi_{1}, \xi_{2} \rightarrow 1$ as $\lambda \rightarrow 0$. Thus, we have obtained

$$
\lim _{\lambda \rightarrow 0} w_{2, \lambda}(v)=\frac{(-1)^{\frac{n+1}{2}}}{a^{2}} \int_{0}^{\infty} \frac{u e^{-v u} d u}{K_{\frac{n-1}{2}}^{2}(u)+\pi^{2} I_{\frac{n-1}{2}}^{2}(u)},
$$

since the passage to the limit under the integral sign is justified by (3.20) below.

Moreover, using the above equations and the asymptotic behavior (3.15) of $I_{\frac{n-1}{2}}$ and $K_{\frac{n-1}{2}}$, we obtain for $u \geqslant 1$ and $0<\lambda \leq a$

$$
\frac{1}{\lambda} \frac{\left[I_{\frac{n-1}{2}}(x u / a) K_{\frac{n-1}{2}}(u)-I_{\frac{n-1}{2}}(u) K_{\frac{n-1}{2}}(x u / a)\right]}{K_{\frac{n-1}{2}}^{2}(u)+\pi^{2} I_{\frac{n-1}{2}}^{2}(u)} e^{-x u / a} e^{u}
$$




$$
\begin{aligned}
& \leqslant \frac{u}{a} \frac{2^{\frac{n-1}{2}}\left[e^{-x u / a} I_{\frac{n-3}{2}}(x u / a) e^{u} K_{\frac{n-1}{2}}(u)+e^{-u} I_{\frac{n-1}{2}}(u) e^{u} K_{\frac{n-3}{2}}(u)\right]}{(x / a)^{\frac{n-1}{2}}\left(K_{\frac{n-1}{2}}^{2}(u)+\pi^{2} I_{\frac{n-1}{2}}^{2}(u)\right)} \\
& \leqslant c u^{2} \frac{\left[1+E_{1}(x u / a)\right]\left[1+E_{2}(u)\right]+\left[1+E_{1}(u)\right]\left[1+E_{2}(u)\right]}{a \cosh (2 u)} \\
(3.19) & \leqslant \frac{C u^{2}}{\cosh (2 u)} .
\end{aligned}
$$

For $u \leqslant 1$ we have

$$
\begin{gathered}
\frac{1}{\lambda} \frac{\left[I_{\frac{n-1}{2}}(x u / a) K_{\frac{n-1}{2}}(u)-I_{\frac{n-1}{2}}(u) K_{\frac{n-1}{2}}(x u / a)\right]}{K_{\frac{n-1}{2}}^{2}(u)+\pi^{2} I_{\frac{n-1}{2}}^{2}(u)} e^{-x u / a} e^{u} \\
\leqslant 2^{\frac{n-1}{2}} \frac{u}{a} \frac{I_{\frac{n-3}{2}}(2 u) K_{\frac{n-1}{2}}(u)+I_{\frac{n-1}{2}}(u) K_{\frac{n-3}{2}}(u)}{K_{\frac{n-1}{2}}^{2}(u)+\pi^{2} I_{\frac{n-1}{2}}^{2}(u)} e^{u}
\end{gathered}
$$

Now, if $\frac{n-1}{2}-1>0$ (i.e. $n>3$ ), using the asymptotics (3.16) we obtain that the above expression is bounded from above by

$$
C u^{n-1} \frac{1+u^{2}}{1+\pi^{2} u^{2 n-2}} \leq \tilde{C} u^{n-1}, \quad u \in(0,1) .
$$

For $\frac{n-1}{2}=1$ (i.e. $n=3$ ) one obtains in fact the same bound

$$
C u^{2} \frac{1+u^{2} \log (2 / u)}{1+u^{4}} \leqslant C u^{2}, \quad u \in(0,1)
$$

Thus, we finally get for $\frac{n-1}{2} \geqslant 1$

$$
w_{2}^{*}(v)=\sup _{0<\lambda \leqslant a}\left|w_{2, \lambda}(v)\right| \leqslant C_{1} \int_{0}^{1} u^{n} e^{-v u} d u+C_{2} \int_{1}^{\infty} \frac{u^{3} e^{-v u} d u}{\cosh (2 u)} .
$$

Now, one easily obtains

$$
w_{2}^{*}(v) \leqslant C(n, a) \quad \text { and } \quad w_{2}^{*}(v) \leqslant C_{1} / v^{n+1}+C_{2} e^{-v}
$$

and the conclusions concerning the function $w_{2}^{*}(v)$ follow.

To finish the proof we show the existence and compute the limit

$$
\lim _{v \rightarrow \infty} v^{n+1} w_{2, \lambda}(v)
$$


As before, we take into account the expression under the integral sign in (3.14) multiplied by $v^{n+1}$ and, after changing variables $t=v u$ we obtain

$$
\begin{aligned}
& \frac{v^{n+1} u\left[I_{\frac{n-1}{2}}(x u / a) K_{\frac{n-1}{2}}(u)-I_{\frac{n-1}{2}}(u) K_{\frac{n-1}{2}}(x u / a)\right]}{K_{\frac{n-1}{2}}^{2}(u)+\pi^{2} I_{\frac{n-1}{2}}^{2}(u)} e^{-\lambda u / a} e^{-v u} d u \\
& =\frac{v^{n-1} t\left[I_{\frac{n-1}{2}}(x t / a v) K_{\frac{n-1}{2}}(t / v)-I_{\frac{n-1}{2}}(t / v) K_{\frac{n-1}{2}}(x t / a v)\right]}{K_{\frac{n-1}{2}}^{2}(t / v)+\pi^{2} I_{\frac{n-1}{2}}^{2}(t / v)} e^{-\lambda t / a v} e^{-t} d t .
\end{aligned}
$$

Using the same formulas (3.16) as before, we obtain that for any fixed $t>0$ the expression above has the following asymptotics when $v \rightarrow \infty$

$$
\begin{aligned}
\frac{v^{n-1} t c_{n} c_{n}^{\prime}\left[(x t / a v)^{\frac{n-1}{2}}(t / v)^{-\frac{n-1}{2}}-(x t / a v)^{-\frac{n-1}{2}}(t / v)^{\frac{n-1}{2}}\right]}{\left(c_{n}^{\prime}\right)^{2}(t / v)^{1-n}+\pi^{2} c_{n}^{2}(t / v)^{n-1}} e^{-\lambda t / a v} e^{-t} & \\
= & \frac{(x / a)^{n-1}-1}{(x / a)^{\frac{n-1}{2}}} \frac{c_{n} c_{n}^{\prime} t^{n}}{\left(c_{n}^{\prime}\right)^{2}+\pi^{2} c_{n}^{2}(t / v)^{2 n-2}} e^{-\lambda t / a v} e^{-t} \\
& \rightarrow \frac{(x / a)^{n-1}-1}{(x / a)^{\frac{n-1}{2}}} \frac{c_{n}}{c_{n}^{\prime}} t^{n} e^{-t} .
\end{aligned}
$$

Observe that, for the sake of simplicity, we wrote here $c_{n}$ and $c_{n}^{\prime}$ instead of $c_{\frac{n-1}{2}}$ and $c_{\frac{n-1}{2}}^{\prime}$. Note that for any fixed $t>0$ and $v$ such that $t<v$ we get that (3.21) is bounded by $c(x, a, n) t^{n} e^{-t}$.

Now, we write

$$
v^{n+1} w_{2, \lambda}(v)=(-1)^{\frac{n+1}{2}} \frac{(x / a)^{\frac{n-1}{2}}}{\lambda a}\left(\int_{0}^{v} \ldots+\int_{v}^{\infty} \ldots\right) .
$$

We use (3.19) with $t / v=u \geqslant 1$ and we observe that the expression in the second integral is bounded from above by

$$
t^{2}(x / a)^{\frac{n-1}{2}} v^{n-2} \frac{\exp (x t / a v-t / v)}{\cosh (2 t / v)} e^{-\lambda t / a v} e^{-t} \leqslant v^{n-2}(x / a)^{-\frac{n-1}{2}} t^{2} e^{-t} .
$$

Since $v \rightarrow \infty$, the second integral tends to 0 , while the first one converges to the following limit

$$
\begin{aligned}
\lim _{v \rightarrow \infty} v^{n+1} w_{2, \lambda}(v) & =(-1)^{\frac{n+1}{2}} \frac{(x / a)^{\frac{n-1}{2}}}{\lambda a} \frac{(x / a)^{n-1}-1}{(x / a)^{\frac{n-1}{2}}} \frac{c_{n}}{c_{n}^{\prime}} \int_{0}^{\infty} t^{n} e^{-t} d t \\
& =(-1)^{\frac{n+1}{2}} n ! \frac{(x / a)^{n-1}-1}{\lambda a} \frac{c_{n}}{c_{n}^{\prime}} .
\end{aligned}
$$

This ends the proof of the theorem. 


\section{Examples.}

We finish this section by writing down explicit integral formulas for some special cases. Observe that in $\mathbb{H}^{2}$ our Poisson kernel is identical with the Euclidean one. Thus, the simplest nontrivial situation arises in $\mathbb{H}^{3}$. Recall that $\lambda=x-a$.

Corollary 3.4 If $n=3$ then

$$
w_{\lambda}(v)=\frac{x}{\lambda a^{2}} \int_{0}^{\infty} \frac{I_{1}(x u / a) K_{1}(u)-K_{1}(x u / a) I_{1}(u)}{K_{1}^{2}(u)+\pi^{2} I_{1}^{2}(u)} e^{-u \lambda / a} e^{-v u} u d u,
$$

with $L(\lambda, y, v)$ defined by

$$
\frac{L(\lambda, y, v)}{\left(\lambda^{2}+|y|^{2}\right)\left((\lambda+a v)^{2}+|y|^{2}\right)^{1 / 2}}=\left(\frac{\lambda^{2}+|y|^{2}}{(\lambda+a v)^{2}+|y|^{2}}\right)^{1 / 2}-1+\frac{1}{2} \frac{a v(2 \lambda+a v)}{\lambda^{2}+|y|^{2}}
$$

hence

$$
P_{a}(x, y)=\frac{\lambda}{2 \pi\left(\lambda^{2}+|y|^{2}\right)^{\frac{3}{2}}} \int_{0}^{\infty} \frac{w_{\lambda}(v) L(\lambda, y, v)}{\left((\lambda+a v)^{2}+|y|^{2}\right)^{\frac{1}{2}}} d v .
$$

If $n=4$ then $w_{\lambda}(v)=a^{-2} e^{-v}$ and

$$
P_{a}(x, y)=\frac{\lambda}{2 \pi^{2}\left(\lambda^{2}+|y|^{2}\right)^{2}} \int_{0}^{\infty} \frac{(2 \lambda+a v)^{2} v^{2} e^{-v}}{(\lambda+a v)^{2}+|y|^{2}} d v
$$

If $n=6$ then

$$
w_{\lambda}(v)=\frac{3}{a^{3}} e^{-3 v / 2}[(2 \lambda+a) \cos (\sqrt{3} v / 2)+\sqrt{3} a \sin (\sqrt{3} v / 2)]
$$

and

$$
L(\lambda, y, v)=(a v(2 \lambda+a v))^{2}\left[2 a v(2 \lambda+a v)+3\left(\lambda^{2}+|y|^{2}\right)\right]
$$

hence

$$
P_{a}(x, y)=\frac{\lambda}{2 \pi^{3}\left(\lambda^{2}+|y|^{2}\right)^{3}} \int_{0}^{\infty} \frac{w_{\lambda}(v) L(\lambda, y, v)}{\left((\lambda+a v)^{2}+|y|^{2}\right)^{2}} d v
$$

Proof. In the case $n=3$ the function $K_{\frac{n-1}{2}}=K_{1}$ has no zeros and we have $w_{\lambda}(v)=w_{2, \lambda}(v)$. We use Theorems 3.2 and 3.3.

For $n=4$ we have $m_{0}(x)=1, m_{1}(x)=2(1+x)$. Certainly, $w_{\lambda}(v)=$ $w_{1, \lambda}(v)$ and $L(\lambda, y, v)=(a v(2 \lambda+a v))^{2}$ so all we have to do is to find the function $w_{\lambda}(v)$. We apply Theorem 3.3 and obtain

$$
\begin{gathered}
w_{\lambda}(v)=w_{1, \lambda}(v)=\frac{-1}{2 \lambda a} \frac{m_{1}(-x / a)}{m_{0}(-1)} e^{-v}=\frac{(-1 / a) 2(1-x / a)}{2 \lambda} e^{-v}=a^{-2} e^{-v} . \\
\text { If } n=6 \text { then } s=2 \text { and } m_{2}(z)=8\left(z^{2}+3 z+3\right)=8\left(z-\frac{-3+i \sqrt{3}}{2}\right)\left(z-\frac{-3-i \sqrt{3}}{2}\right) .
\end{gathered}
$$


Put $z_{1}=-3 / 2+i \sqrt{3} / 2$. According to formula (3.18) we obtain

$$
\begin{aligned}
\operatorname{Res}_{z_{1}} F_{\lambda} & =\frac{-1}{(6-2) \lambda a} \frac{m_{2}\left(x z_{1} / a\right)}{m_{1}\left(z_{1}\right)}=\frac{-1}{4 \lambda a} \frac{8\left(\left(x z_{1} / a\right)^{2}+3\left(x z_{1} / a\right)+3\right)}{2\left(1+z_{1}\right)} \\
& =\frac{-1}{\lambda a}\left(\left(x z_{1} / a\right)^{2}+3\left(x z_{1} / a\right)+3\right)\left(1+\overline{z_{1}}\right) \\
& =\frac{-1}{\lambda a}\left(\left(x z_{1} / a\right)^{2}-z_{1}^{2}+3\left(x z_{1} / a\right)-3 z_{1}\right)\left(1+\overline{z_{1}}\right) \\
& =\frac{-1}{a^{2}}\left[(1+x / a) z_{1}^{2}\left(1+\overline{z_{1}}\right)+3 z_{1}\left(1+\overline{z_{1}}\right)\right]=\frac{3}{2 a^{3}}[2 \lambda+a-i \sqrt{3} a] .
\end{aligned}
$$

Finally, we have

$$
\begin{aligned}
w_{\lambda}(v) & =w_{1, \lambda}(v)=\frac{3 e^{-3 v / 2}}{a^{3}} \Re\left([2 \lambda+a-i \sqrt{3} a] e^{i \sqrt{3} v / 2}\right) \\
& =\frac{3 e^{-3 v / 2}}{a^{3}}[(2 \lambda+a) \cos (\sqrt{3} v / 2)+\sqrt{3} a \sin (\sqrt{3} v / 2)] .
\end{aligned}
$$

This completes the case when $n=6$.

\section{Asymptotic behaviour}

In this section we study the asymptotic behaviour of the Poisson kernel $P_{a}(x, y)$. The hardest part is to get the asymptotics for $|y| \rightarrow \infty$ (see Theorem 4.9 below). It is clear that for integrals like (2.5) the Lebesgue bounded convergence theorem fails. Another natural approach by a Tauberian theorem (or the Karamata theory) does not lead to the solution either. The main obstacle is that at the Laplace transform level, while taking limits, we have to deal with fine cancellations of divergent integrals which is difficult (if not impossible) to control. Our representation formula in the basic cases $n=4$ or $n=6$ gives almost immediately the required asymptotics which indicates the advantage of the presented approach and was an inspiration for the remaining part of the work.

We shall compare our results to the behaviour of the classical Poisson kernel of the upper half-space in $\mathbb{R}^{n}$,

$$
P_{\mathbb{R}^{n}}(x, y)=\frac{\Gamma(n / 2)}{\pi^{n / 2}} \frac{x}{\left(x^{2}+|y|^{2}\right)^{n / 2}}, \quad x>0, \quad y \in \mathbb{R}^{n-1},
$$

and the Poisson kernel of the entire hyperbolic space in half-space model,

$$
P_{\mathbb{H}^{n}}(x, y)=\frac{\Gamma(n-1)}{\pi^{\frac{n-1}{2}} \Gamma\left(\frac{n-1}{2}\right)}\left(\frac{x}{x^{2}+|y|^{2}}\right)^{n-1}, \quad x>0, \quad y \in \mathbb{R}^{n-1},
$$

see [11], [12]. 
The constant in the last formula is easily determined knowing that

$$
\int_{\mathbb{R}^{n-1}} P_{\mathbb{H}^{n}}(x, y) d y=1
$$

on one side and that, on the other side,

$$
\begin{aligned}
\int_{\mathbb{R}^{n-1}}\left(\frac{1}{1+|y|^{2}}\right)^{n-1} d y & =\frac{2 \pi^{\frac{n-1}{2}}}{\Gamma\left(\frac{n-1}{2}\right)} \int_{0}^{\infty} \frac{r^{n-2}}{\left(1+r^{2}\right)^{n-1}} d r \\
& =\frac{\pi^{\frac{n-1}{2}}}{\Gamma\left(\frac{n-1}{2}\right)} B\left(\frac{n-1}{2}, \frac{n-1}{2}\right)
\end{aligned}
$$

according to $[10,3.194 .3]$.

In the two particular cases of Propositions 4.4 and 4.5 all the three Poisson kernels behave in the same way.

The main tools in our study of the asymptotics of $P_{a}(x, y)$ are the representation formula (3.7) from Theorem 3.2 and the semigroup properties of the Poisson kernel.

Semigroup property of $P_{a}(x, \cdot)$.

By the strong Markov property we obtain the following semigroup property of $P_{a}(x, y)$.

Proposition 4.1 Let $b$ be such that $0<a<b<x$. Then

$$
P_{a}(x, y)=\int_{\mathbb{R}^{n-1}} P_{b}(x, z) P_{a}(b, y-z) d z, \quad y \in \mathbb{R}^{n-1}
$$

Denoting $P_{a, x}(y)=P_{a}(x, y)$ we have

$$
P_{a, x}=P_{a, b} * P_{b, x}, \quad 0<a<b<x,
$$

where $*$ is the usual convolution in $\mathbb{R}^{n-1}$.

Proof. Observe that $\tau_{b}<\tau_{a}$ so using the strong Markov property we obtain for an arbitrary nonnegative and bounded Borel measurable function $f$ on $\mathbb{R}^{n-1}$ :

$$
\begin{aligned}
E^{x} f\left(X_{\tau_{a}}\right) & =E^{x} E^{X_{\tau_{b}}}\left[f\left(X_{\tau_{a}}\right)\right] \\
& =\int_{\mathbb{R}^{n-1}} P_{b}(x, z)\left\{\int_{\mathbb{R}^{n-1}} P_{a}(z, y) f(y) d y\right\} d z \\
& =\int_{\mathbb{R}^{n-1}} f(y)\left\{\int_{\mathbb{R}^{n-1}} P_{b}(x, z) P_{a}(b, y-z) d z\right\} d y
\end{aligned}
$$


Thus, we obtain that almost everywhere the following holds

$$
P_{a}(x, y)=\int_{\mathbb{R}^{n-1}} P_{b}(x, z) P_{a}((z, b), y) d z,
$$

where we denote $P_{a}(x, y)=P_{a}((0, \ldots, 0, x), y)$ and, according to this notation, we also have $P_{a}((z, b), y)=P_{a}(b, y-z)$. Since both sides of the above equation are continuous as a function of $y$, the formula (4.2) follows.

Remark. The semigroup formula (4.2) holds also for $a=0$, with $P_{0, x}(y)=$ $P_{\mathbb{H}^{n}}(x, y)$. This follows from the fact that as in [1], $P_{\mathbb{H}^{n}}$ is the density of $\tilde{X}_{\infty}(x)$, and the proof of Proposition 4.1 still works in this case.

Moreover, when $a \rightarrow b, a>b$ or when $b \rightarrow a, b>a$, then $P_{a, b} \Rightarrow \delta_{0}$. Consequently, $\left\{P_{a, b}\right\}$ is a $2-$ parameter continuous probability semigroup. It means that $P_{a, b}$ are the densities of the increments $Y_{b}-Y_{a}$ of a nonhomogeneous Lévy process $\left\{Y_{x}\right\}_{0<x<\infty}$, with the distribution of $Y_{x}$ equal to $\tilde{X}_{\infty}(x)$ in $\mathbb{R}^{n-1}$.

\section{Asymptotics when $a \rightarrow 0$.}

When the boundary of the half-space in the Euclidean space $\mathbb{R}^{n}$ is moving away to $-\infty$, the Poisson kernel converges to 0 . This is not the case in hyperbolic spaces. In $\mathbb{H}^{n}$ we will show the uniform convergence of $P_{a}(x, \cdot)$ to the Poisson kernel of $\mathbb{H}^{n}$, given by (4.1).

Note that the weak convergence, equivalent to the pointwise convergence of the Fourier transforms, is simple to see by a probabilistic argument using $X_{\tau_{a}} \Rightarrow X_{\infty}$. An easy analytic proof of the pointwise convergence of the Fourier transforms is based on Theorem 2.1, on the asymptotics $v^{\nu} K_{\nu}(v) \sim$ $2^{\nu-1} \Gamma(\nu), \quad v \rightarrow 0$, and on the fact that

$$
\mathcal{F}\left[P_{\mathbb{H}^{n}}(x, \cdot)\right](u)=\frac{1}{2^{\frac{n-3}{2}} \Gamma\left(\frac{n-1}{2}\right)}(x|u|)^{\frac{n-1}{2}} K_{\frac{n-1}{2}}(x|u|) .
$$

The last formula follows e.g. from [10, 6.576.7]:

$$
\int_{0}^{\infty} r^{\mu+\nu+1} J_{\mu}(r|y|) K_{\nu}(r x) d r=2^{\mu+\nu}|y|^{\mu} x^{\nu} \frac{\Gamma(\mu+\nu+1)}{\left(x^{2}+|y|^{2}\right)^{\mu+\nu+1}}
$$

with $\mu>\nu-1, x>0$. Putting $\nu=\frac{n-1}{2}, \mu=\frac{n-3}{2}+\epsilon(\epsilon>0)$, we have $\mu+\nu+1=n-1+\epsilon$. Taking limit $\epsilon \rightarrow 0$, by the dominated convergence theorem we easily extend (4.3) to the special case $\mu=\nu-1$.

Proposition 4.2 Let $n \in \mathbb{N}, n \geq 2$. Then for all $y \in \mathbb{R}^{n-1}$ and $x>0$ we have

$$
\lim _{a \rightarrow 0} P_{a}(x, y)=P_{\mathbb{H}^{n}}(x, y)
$$

and the convergence is uniform with respect to $y \in \mathbb{R}^{n-1}$. 
Proof. We have, by elementary properties of the convolution, for any $a, b$ such that $0<a<b<x$

$$
\left\|P_{a, x}-P_{0, x}\right\|_{\infty}=\left\|P_{a, b} * P_{b, x}-P_{0, a} * P_{a, b} * P_{b, x}\right\|_{\infty} \leqslant\left\|P_{0, a} * P_{b, x}-P_{b, x}\right\|_{\infty} .
$$

Note that $P_{0, a} * P_{b, x}$ is the action of a probabilistic operator $T_{0, a}$ with density $P_{0, a}$ on the continuous function $P_{b, x}$. The function $P_{b, x}$ is bounded by Theorem 2.2.

The operators $T_{a, x}$ form a continuous 2-parameter semigroup, so

$$
\lim _{a \rightarrow 0}\left\|T_{0, a} g-g\right\|_{\infty}=0,
$$

for any continuous bounded function $g$. Thus

$$
\left\|P_{0, a} * P_{b, x}-P_{b, x}\right\|_{\infty} \rightarrow 0, \quad a \rightarrow 0,
$$

and the assertion of the proposition follows.

A different proof of Proposition 4.2 is also possible, by justifying the passage with $a \rightarrow 0$ under the integral in (2.5) and by the Lebesgue bounded convergence theorem.

Proposition 4.2 implies the following limit theorem for the hyperbolic Brownian motion.

Corollary 4.3 Let $\mathbf{x}=\left(x_{i}\right)_{i=1, \ldots, n} \in \mathbb{H}^{n}$ and $X_{t}$ be the hyperbolic Brownian motion starting at $\mathbf{x}$. Then $X_{\tau_{a}}$, the process $X_{t}$ stopped when first crossing the hyperplane $\left\{y_{n}=a\right\}$, converges when $a \rightarrow 0$ to a random variable $X_{\infty}$, concentrated on the boundary $\left\{y_{n}=0\right\}$ of $\mathbb{H}^{n}$ and with the density

$$
P_{0}(\mathbf{x}, y)=\frac{\Gamma(n-1)}{\pi^{\frac{n-1}{2}} \Gamma\left(\frac{n-1}{2}\right)}\left(\frac{x_{n}}{x_{n}^{2}+|y-\tilde{\mathbf{x}}|^{2}}\right)^{n-1},
$$

where $\tilde{\mathbf{x}}=\left(x_{1}, \ldots, x_{n-1}\right)$. The convergence of $X_{\tau_{a}}$ to $X_{\infty}$ is in the sense of uniform convergence of the densities of their distributions, when we project the hyperplanes $\left\{y_{n}=a\right\}$ onto the boundary $\left\{y_{n}=0\right\}$.

Remark. By Scheffe's theorem, the distributions of $\tilde{X}_{\tau_{a}}$ converge to the distribution of $X_{\infty}$ in the total variation norm.

Asymptotics when $x \rightarrow \infty$.

The Poisson kernel $P_{a}(x, y)$ behaves in the same way as the Euclidean Poisson kernel and the Poisson kernel of $\mathbb{H}^{n}$ :

Proposition 4.4 We have

$$
P_{a}(x, y) \sim c x^{-n+1}, \quad x \rightarrow \infty .
$$


Proof. Observe that for $n \geq 2$

$$
(a / x)^{\frac{n-1}{2}} \leq e^{r(x-a)} \frac{K_{\frac{n-1}{2}}(r x)}{K_{\frac{n-1}{2}}(r a)} \leq(a / x)^{\frac{1}{2}}, \quad r>0 .
$$

Indeed, since (cf. [10, 8.432.8])

$$
K_{\frac{n-1}{2}}(z)=\frac{\Gamma\left(\frac{1}{2}\right)}{\Gamma\left(\frac{n}{2}\right)}\left(\frac{z}{2}\right)^{\frac{n-1}{2}} e^{-z} \int_{0}^{\infty} e^{-z u}(u+2)^{\frac{n}{2}-1} u^{\frac{n}{2}-1} d u
$$

by the change of variable $\bar{u}=r x u(\bar{u}=r a u$, respectively) we get

$$
e^{r(x-a)} \frac{K_{\frac{n-1}{2}}(r x)}{K_{\frac{n-1}{2}}(r a)}=\left(\frac{a}{x}\right)^{\frac{1}{2}} \frac{\int_{0}^{\infty} e^{-u}(u /(r x)+2)^{\frac{n-2}{2}} u^{\frac{n-2}{2}} d u}{\int_{0}^{\infty} e^{-u}(u /(r a)+2)^{\frac{n-2}{2}} u^{\frac{n-2}{2}} d u} .
$$

Since $x>a$ the above quotient of integrals does not exceed 1 and we get the upper bound in (4.4). Multiplying the left-hand side of (4.5) by

$$
1=\left(\frac{a}{x}\right)^{\frac{n-2}{2}} \frac{(r x)^{\frac{n-2}{2}}}{(r a)^{\frac{n-2}{2}}}
$$

we get

$$
e^{r(x-a)} \frac{K_{\frac{n-1}{2}}(r x)}{K_{\frac{n-1}{2}}(r a)}=\left(\frac{a}{x}\right)^{\frac{n-1}{2}} \frac{\int_{0}^{\infty} e^{-u}(u+2 r x)^{\frac{n-2}{2}} u^{\frac{n-2}{2}} d u}{\int_{0}^{\infty} e^{-u}(u+2 r a)^{\frac{n-2}{2}} u^{\frac{n-2}{2}} d u} .
$$

Now, $x>a$ implies that the above quotient of the integrals is greater than 1 and the lower bound in (4.4) is verified.

First, we deal with the special case $y=0$. By a simple change of variable $r x=t$ in (2.6) and by (4.6) we get

$$
\begin{aligned}
P_{a}(x, 0) & =c\left(\frac{x}{a}\right)^{\frac{n-1}{2}} \frac{1}{x^{n-1}} \int_{0}^{\infty} \frac{K_{\frac{n-1}{2}}(t)}{K_{\frac{n-1}{2}}(t a / x)} t^{n-2} d t \\
& =\frac{c}{x^{n-1}} \int_{0}^{\infty} e^{-t(1-a / x)} \frac{\int_{0}^{\infty} e^{-u}(u+2 t)^{\frac{n-2}{2}} u^{\frac{n-2}{2}} d u}{\int_{0}^{\infty} e^{-u}(u+2 t a / x)^{\frac{n-2}{2}} u^{\frac{n-2}{2}} d u} t^{n-2} d t .
\end{aligned}
$$

For each $t>0$, when $x$ increases to infinity, the denominator decreases to $\int_{0}^{\infty} e^{-u} u^{n-2} d u=\Gamma(n-1)$. Hence, for $x>2 a$ we have

$$
\frac{e^{-t(1-a / x)}}{\int_{0}^{\infty} e^{-u}(u+2 t a / x)^{\frac{n-2}{2}} u^{\frac{n-2}{2}} d u} \leq \frac{e^{-t / 2}}{\Gamma(n-1)} .
$$

Therefore, by bounded convergence theorem the assertion for $|y|=0$ follows. 
Now, assume $|y|>0$. Recall that for $\nu>0$

$$
K_{\nu}(z) \sim 2^{\nu-1} \Gamma(\nu) z^{-\nu}, \quad z \rightarrow 0 .
$$

By a simple change of variable $r x=z$ in $(2.5)$ we get

$$
\begin{aligned}
P_{a}(x, y) & =c \frac{|y|^{-\frac{n-3}{2}}}{x a^{\frac{n-1}{2}}} \int_{0}^{\infty} \frac{K_{\frac{n-1}{2}}(z)}{K_{\frac{n-1}{2}}(z a / x)} J_{\frac{n-3}{2}}(z|y| / x) z^{\frac{n-1}{2}} d z \\
= & c x^{-n+1} \int_{0}^{\infty} K_{\frac{n-1}{2}}(z) \frac{x^{\frac{n-1}{2}}}{(z a)^{\frac{n-1}{2}} K_{\frac{n-1}{2}}(z a / x)} \frac{x^{\frac{n-3}{2}} J_{\frac{n-3}{2}}(z|y| / x)}{(z|y|)^{\frac{n-3}{2}}} z^{\frac{3 n-5}{2}} d z .
\end{aligned}
$$

By (4.7) and (2.8) the two quotients above converge to a positive constant when $x \rightarrow \infty$. Moreover, the second one remains uniformly bounded in $z \in(0, \infty)$ and $x>0$. For $z<x / a$ by (4.7) we get

$$
K_{\frac{n-1}{2}}(z) z^{\frac{3 n-5}{2}} \frac{x^{\frac{n-1}{2}}}{(z a)^{\frac{n-1}{2}} K_{\frac{n-1}{2}}\left(\frac{z a}{x}\right)} \leq c K_{\frac{n-1}{2}}(z) z^{\frac{3 n-5}{2}} .
$$

Using (3.15), for $z>x / a$ and $x>2 a$, say, we get

$$
\begin{aligned}
K_{\frac{n-1}{2}}(z) z^{\frac{3 n-5}{2}} \frac{x^{\frac{n-1}{2}}}{(z a)^{\frac{n-1}{2}} K_{\frac{n-1}{2}}\left(\frac{z a}{x}\right)} & \leq c\left(\frac{x}{a}\right)^{\frac{n}{2}} \frac{z^{\frac{3 n-5}{2}}}{z^{\frac{n-1}{2}}} \exp (-z(1-a / x)) \\
& \leq c z^{\frac{3 n-4}{2}} \exp (-z / 2) .
\end{aligned}
$$

By this and (4.8) bounded convergence theorem applies. Consequently, the whole integral above tends to a positive constant as $x \rightarrow \infty$. The assertion follows.

\section{Asymptotics when $x \rightarrow a$.}

The following asymptotic is elementary.

\section{Proposition 4.5}

$$
P_{a}(x, 0) \sim \frac{2^{2-n} \Gamma(n-1) \pi^{-\frac{n-1}{2}}}{\Gamma\left(\frac{n-1}{2}\right)}(x-a)^{-n+1}, \quad x \rightarrow a^{+} .
$$

Proof. From (4.4) it follows that

$$
\begin{aligned}
\Gamma(n-1) & (a / x)^{\frac{n-1}{2}}(x-a)^{-n+1}=(a / x)^{\frac{n-1}{2}} \int_{0}^{\infty} e^{-r(x-a)} r^{n-2} d r \\
& \leq \int_{0}^{\infty} \frac{K_{\frac{n-1}{2}}(r x)}{K_{\frac{n-1}{2}}(r a)} r^{n-2} d r \leq\left(\frac{a}{x}\right)^{\frac{1}{2}} \int_{0}^{\infty} e^{-r(x-a)} r^{n-2} d r \\
& =\Gamma(n-1)(a / x)^{\frac{1}{2}}(x-a)^{-n+1} .
\end{aligned}
$$

Combining this and (2.6) completes the proof. 
Much more is required, however, to obtain the following Euclidean-like asymptotics

$$
P_{a}(x, y) \sim c(x-a), \quad x \rightarrow a^{+}, \quad|y| \neq 0 .
$$

The justification of this important result is postponed after the proof of Theorem 4.9 .

Asymptotics when $|y| \rightarrow \infty$.

The most important and difficult to prove is what happens when $|y| \rightarrow \infty$. By $n$ we denote, as before, the dimension of the considered hyperbolic space $\mathbb{H}^{n}$. Recall that $\lambda=x-a$. We assume throughout this section that $n>2$. Let us rewrite the basic formula for $P_{a}(x, y)$, using some notation more suitable for calculations. Denote

$$
\begin{gathered}
\kappa=(\lambda+a v)^{2}-\lambda^{2}, \\
\Phi(u)=\Phi_{\frac{n}{2}-1}(u)=(1+u)^{1-\frac{n}{2}}-1+\left(\frac{n}{2}-1\right) u, \quad u \geqslant 0 .
\end{gathered}
$$

We then have $(\lambda+a v)^{2}+|y|^{2}=\kappa+\lambda^{2}+|y|^{2}$, so the formula (3.8) takes the form:

$$
\begin{aligned}
& \frac{L(\lambda, y, v)}{\left(\lambda^{2}+|y|^{2}\right)\left(\kappa+\lambda^{2}+|y|^{2}\right)^{\frac{n}{2}-1}} \\
& =\left(\frac{n}{2}-1\right) \frac{\kappa}{\lambda^{2}+|y|^{2}}-1+\left(\frac{\lambda^{2}+|y|^{2}}{\kappa+\lambda^{2}+|y|^{2}}\right)^{\frac{n}{2}-1} \\
& =\left(1+\frac{\kappa}{\lambda^{2}+|y|^{2}}\right)^{1-\frac{n}{2}}-1+\left(\frac{n}{2}-1\right) \frac{\kappa}{\lambda^{2}+|y|^{2}} \\
& =\Phi\left(\frac{\kappa}{\lambda^{2}+|y|^{2}}\right) .
\end{aligned}
$$

Consequently, according to the formula for the Poisson kernel (3.7) we thus obtain

$$
P_{a}(x, y)=\frac{\Gamma\left(\frac{n}{2}-1\right)}{2 \pi^{n / 2}} \frac{\lambda}{\left(\lambda^{2}+|y|^{2}\right)^{\frac{n}{2}-1}} \int_{0}^{\infty} w_{\lambda}(v) \Phi\left(\frac{\kappa}{\lambda^{2}+|y|^{2}}\right) d v .
$$

Writing the last formula in the form

$$
P_{a}(x, y)=\frac{\Gamma\left(\frac{n}{2}-1\right)}{2 \pi^{n / 2}}|y|^{-n+2} \frac{\lambda}{\left((\lambda /|y|)^{2}+1\right)^{\frac{n}{2}-1}} \int_{0}^{\infty} w_{\lambda}(v) \Phi\left(\frac{\kappa}{\lambda^{2}+|y|^{2}}\right) d v
$$

we see that in order to get the asymptotics of $P_{a}(x, y)$ when $|y| \rightarrow \infty$, it is sufficient to obtain the asymptotics of

$$
\int_{0}^{\infty} w_{\lambda}(v) \Phi\left(\frac{\kappa}{\lambda^{2}+|y|^{2}}\right) d v
$$

when $|y| \rightarrow \infty$. 
In the sequel we use the following standard properties of the oscillating binomial series

$$
\sum_{j=0}^{\infty}(-1)^{j} \frac{\left(\frac{n}{2}-1\right)_{j}}{j !} u^{j}, \quad\left(\frac{n}{2}-1\right)_{j}=\left(\frac{n}{2}-1\right) \frac{n}{2} \ldots\left(\frac{n}{2}+j-2\right),
$$

related to the function $\Phi$. For all $u \geqslant 0$ and $l \geqslant 2$ we have

$$
\left|\Phi(u)-\sum_{2 \leqslant j \leqslant l-1}(-1)^{j} \frac{\left(\frac{n}{2}-1\right)_{j}}{j !} u^{j}\right| \leqslant \frac{\left(\frac{n}{2}-1\right)_{l}}{l !} u^{l} .
$$

Moreover, when $u \rightarrow 0+$,

$$
\lim _{u \rightarrow 0+} u^{-l}\left[\Phi(u)-\sum_{2 \leqslant j \leqslant l-1}(-1)^{j} \frac{\left(\frac{n}{2}-1\right)_{j}}{j !} u^{j}\right]=(-1)^{l} \frac{\left(\frac{n}{2}-1\right)_{l}}{l !} .
$$

We also have for all $u \geqslant 0$

$$
(-1)^{l}\left[\Phi(u)-\sum_{2 \leqslant j \leqslant l-1}(-1)^{j} \frac{\left(\frac{n}{2}-1\right)_{j}}{j !} u^{j}\right] \geqslant 0 .
$$

Note that for $l=2$ the formulas above involve the function $\Phi$ alone (summation is performed over empty set of indices).

Before the formal proof of the asymptotics of the Poisson kernel as $|y| \rightarrow \infty$ we first consider what happens on $\mathbb{H}^{4}$ and $\mathbb{H}^{6}$.

On $\mathbb{H}^{4}$ the situation is quite clear. Taking into account the explicit formula for the Poisson kernel computed in the end of Section 3 we see that the asymptotics is of order $|y|^{-6}$. On $\mathbb{H}^{6}$ we encounter more delicate situation. We have $L(\lambda, y, v)=(a v(2 \lambda+a v))^{2}\left[2 a v(2 \lambda+a v)+3\left(\lambda^{2}+|y|^{2}\right)\right]$, so, together with the formula for the Poisson kernel, one may expect that the precise rate of convergence to 0 on $\mathbb{H}^{6}$ is of order $|y|^{-8}$. However, it turns out that the correct rate is better, namely $|y|^{-10}$ ! To explain what is behind this phenomenon we first show that for all $\lambda>0$

$$
\int_{0}^{\infty}\left((\lambda+a v)^{2}-\lambda^{2}\right)^{2} w_{\lambda}(v) d v=\int_{0}^{\infty} \kappa^{2} w_{\lambda}(v) d v=0
$$

To prove this we recall from the proof of Theorem 3.2 the following formula:

$$
\lambda F_{\lambda}(r a)=r \frac{m_{2}(r x)}{m_{2}(r a)}-\left(\frac{x}{a}\right)^{2}\left(r-3 \frac{\lambda}{a x}\right),
$$

where

$$
F_{\lambda}(r)=\int_{0}^{\infty} e^{-r v} w_{\lambda}(v) d v, \quad \text { and } \quad m_{2}(x)=8\left(x^{2}+3 x+3\right) .
$$


Now, by the direct differentiation of the equation defining $F_{\lambda}(r a)$ we obtain

$$
\begin{aligned}
F_{\lambda}^{\prime \prime}(0) & =\int_{0}^{\infty} v^{2} w_{\lambda}(v) d v=\frac{2}{a^{2}} \\
F_{\lambda}^{\prime \prime \prime}(0) & =-\int_{0}^{\infty} v^{3} w_{\lambda}(v) d v=\frac{2(\lambda-a)}{a^{3}} \\
F_{\lambda}^{(i v)}(0) & =\int_{0}^{\infty} v^{4} w_{\lambda}(v) d v=-8 \frac{\lambda}{a^{3}} .
\end{aligned}
$$

With these formulas in hand we justify our claim (4.14) as follows:

$$
\begin{aligned}
\int_{0}^{\infty} \kappa^{2} w_{\lambda}(v) d v & =a^{2}\left[4 \lambda F_{\lambda}^{\prime \prime}(0)-4 a \lambda F_{\lambda}^{\prime \prime \prime}(0)+a^{2} F_{\lambda}^{(i v)}(0)\right] \\
& =a^{2}\left[4 \lambda^{2} \frac{2}{a^{2}}-4 a \lambda \frac{2}{a^{3}}(\lambda-a)-a^{2} 8 \frac{\lambda}{a^{3}}\right]=0 .
\end{aligned}
$$

Taking into account the form of the Poisson kernel and (4.14) we obtain

$$
\begin{aligned}
P_{a}(x, y)= \\
=\frac{\lambda}{2 \pi^{3}\left(\lambda^{2}+|y|^{2}\right)^{2}} \int_{0}^{\infty}\left[\left(1+\frac{\kappa}{\lambda^{2}+|y|^{2}}\right)^{-2}-1+2 \frac{\kappa}{\lambda^{2}+|y|^{2}}\right] w_{\lambda}(v) d v \\
=\frac{\lambda}{2 \pi^{3}\left(\lambda^{2}+|y|^{2}\right)^{2}} \\
\quad \times \int_{0}^{\infty}\left[\left(1+\frac{\kappa}{\lambda^{2}+|y|^{2}}\right)^{-2}-1+2 \frac{\kappa}{\lambda^{2}+|y|^{2}}-3 \frac{\kappa^{2}}{\left(\lambda^{2}+|y|^{2}\right)^{2}}\right] w_{\lambda}(v) d v
\end{aligned}
$$

Taking into account the property (4.12), as well as integrability properties of $w_{\lambda}$ we infer that for fixed $\lambda>0$

$$
\lim _{|y| \rightarrow \infty}\left(\lambda^{2}+|y|^{2}\right)^{5} P_{a}(x, y)=-\frac{2 \lambda}{\pi^{3}} \int_{0}^{\infty} \kappa^{3} w_{\lambda}(v) d v .
$$

If we now show that

$$
\int_{0}^{\infty} \kappa^{3} w_{\lambda}(v) d v \neq 0
$$

this will give us the required rate of convergence to 0 . Now, let us mention that although it is possible (with a considerable effort) to show the property (4.14) directly as above for all spaces $\mathbb{H}^{n}$, with $n>4$, this property alone does not amounts yet for the correct rate if $n$ is large. Indeed, to prove the correct rate of convergence we need much more, namely the following 
Claim. For all hyperbolic spaces of dimension $n>3$ and all $\lambda>0$ we have the following

$$
\int_{0}^{\infty} \kappa^{m} w_{\lambda}(v) d v=0
$$

for all $m$ such that $2 \leqslant m<[(n+1) / 2]$. Moreover, for $m=[(n+1) / 2]$ and $n$ even the integral above is different from 0 while for $n$ odd it is infinite.

Once the above claim is verified, we obtain the following representation of the Poisson kernel, stated here for the sake of convenience:

\section{Corollary 4.6}

$$
\begin{aligned}
& P_{a}(x, y)=\frac{\lambda \Gamma\left(\frac{n}{2}-1\right)}{2 \pi^{n / 2}\left(\lambda^{2}+|y|^{2}\right)^{\frac{n}{2}-1}} \\
& \quad \times \int_{0}^{\infty}\left[\left(1+\frac{\kappa}{\lambda^{2}+|y|^{2}}\right)^{1-\frac{n}{2}}-\sum_{0}^{\left[\frac{n+1}{2}\right]-1}(-1)^{j} \frac{\left(\frac{n}{2}-1\right)_{j}}{j !} \frac{\kappa^{j}}{\left(\lambda^{2}+|y|^{2}\right)^{j}}\right] w_{\lambda}(v) d v .
\end{aligned}
$$

The validity of the above corollary is a direct consequence of Lemma 4.8. For hyperbolic spaces of even dimension this form of the Poisson kernel, together with the second part of the above claim, yields at once the desired convergence rate. If the dimension is odd we additionally have to overcome some integrability problems, stemming out of the asymptotics of the function $w_{\lambda}$ (clarified in Theorem 3.3).

Computational proof of the above claim is beyond our reach. However, this is the place where the semigroup property of the Poisson kernel comes in handy.

The proof of the general case is contained in a series of lemmas. The first one follows from the formula (4.12), if we take into account the integrability properties of the function $w_{\lambda}(v)$.

Lemma 4.7 Let $2 \leqslant l \leqslant[n / 2]$. Then

$$
\begin{gathered}
\lim _{|y| \rightarrow \infty}|y|^{2 l} \int_{0}^{\infty} w_{\lambda}(v)\left[\Phi\left(\frac{\kappa}{\lambda^{2}+|y|^{2}}\right)-\sum_{2 \leqslant j \leqslant l-1}(-1)^{j} \frac{\left(\frac{n}{2}-1\right)_{j}}{j !}\left(\frac{\kappa}{\lambda^{2}+|y|^{2}}\right)^{j}\right] d v \\
=(-1)^{l} \frac{\left(\frac{n}{2}-1\right)_{l}}{l !} \int_{0}^{\infty} \kappa^{l} w_{\lambda}(v) d v .
\end{gathered}
$$

When $n$ is even, this is true for any $l \in \mathbb{N}, l \geqslant 2$. 
Proof. Denote $u=\kappa /\left(\lambda^{2}+|y|^{2}\right)$. We apply the formula (4.12) for the above $u=u(x, a, y, v)$.

We multiply and divide by $u^{l}$ the expression under the integral in the last formula. When $|y| \rightarrow \infty$ then $u \rightarrow 0$, so the formula (4.12) applies to

$$
u^{-l}\left[\Phi(u)-\sum_{2 \leqslant j \leqslant l-1}(-1)^{j} \frac{\left(\frac{n}{2}-1\right)_{j}}{j !} u^{j}\right] .
$$

On the other hand, $|y|^{2 l} u^{l} \rightarrow \kappa^{l}$. The passage to the limit under the integral sign is justified by (4.11) and the fact that

$$
\begin{aligned}
& \int_{0}^{\infty} \kappa^{\left[\frac{n}{2}\right]}\left|w_{\lambda}(v)\right| d v<\infty, \quad n \text { odd }, \\
& \int_{0}^{\infty} \kappa^{m}\left|w_{\lambda}(v)\right| d v<\infty, \quad m \in \mathbb{N}, n \text { even } .
\end{aligned}
$$

Actually, according to Theorem 3.3, when $n$ is even, the function $w_{\lambda}$ has finite moments of all orders and when $n=2 k+1, k=[n / 2]$, the function $v^{n-1} w_{\lambda}(v) \sim \kappa^{k} w_{\lambda}(v)$ is integrable.

The observation contained in the next lemma is crucial for our purposes. It consists of comparing the rate of convergence to zero of our Poisson kernel $P_{a}(x, y)$, as $|y| \rightarrow \infty$, with the corresponding rate of convergence of the global kernel.

Lemma 4.8 For hyperbolic spaces of even dimension $n=2 k>4$ we have for all $\lambda>0$

$$
\int_{0}^{\infty} \kappa^{j} w_{\lambda}(v) d v=0
$$

when $j=2, \ldots, k-1$.

For hyperbolic spaces of odd dimension $n=2 k+1>3$ we have for all $\lambda>0$

$$
\int_{0}^{\infty} \kappa^{j} w_{\lambda}(v) d v=0
$$

when $j=2, \ldots, k$.

Proof. Consider first the case $n=2 k>4$ and suppose that the assertion is false. Let $j_{o} \leqslant k-1$ be the smallest power such that (4.15) does not hold. By Lemma 4.7 and the formula (4.10), it follows that there exists

$$
\lim _{|y| \rightarrow \infty}|y|^{n-2+2 j_{o}} P_{a}(x, y)>0 .
$$


We will show that this is contradictory with the existence of a finite limit

$$
\lim _{|y| \rightarrow \infty}|y|^{2 n-2} P_{0}(x, y) .
$$

By the semigroup property proved in Proposition 4.1 (see Remark below its proof) we have

$$
P_{0}(x, \cdot)=P_{0}(a, \cdot) * P_{a}(x, \cdot) .
$$

It follows that for $|y|>M>0$

$$
\begin{aligned}
|y|^{2 n-2} P_{0}(x, y) & \geqslant\left(\frac{|y|}{|y|+1}\right)^{2 n-2} \int_{|z| \leqslant 1} P_{0}(a, z) P_{a}(x, y-z)|y-z|^{2 n-2} d z \\
& =\left(\frac{|y|}{|y|+1}\right)^{2 n-2} \int_{|z| \leqslant 1} P_{0}(a, z) P_{a}(x, y-z)|y-z|^{n-2+2 j_{o}}|y-z|^{n-2 j_{o}} d z \\
& \geqslant c\left(\frac{|y|}{|y|+1}\right)^{2 n-2} \int_{|z| \leqslant 1} P_{0}(a, z)|y-z|^{n-2 j_{o}} d z \\
& \geqslant\left. c_{1}(|y|-1)\right|^{n-2 j_{o}} \rightarrow \infty, \quad|y| \rightarrow \infty
\end{aligned}
$$

because $n-2 j_{o} \geqslant 2$.

In the case $n=2 k+1>3$, let us remark that Lemma 4.7 applies for $l \leqslant k$. We proceed exactly in the same way as in the proof in the case of $n$ even, with the only difference that now $j_{o} \leqslant k$, so $n-2 j_{o} \geqslant 1$ and the final contradiction with $\lim _{|y| \rightarrow \infty}|y|^{2 n-2} P_{0}(x, y)<\infty$ also holds.

Let us point out once again that the result of Lemma 4.8 enables us to write our Poisson kernel in the form indicated in Corollary 4.6. Corollary 4.6, together with Lemma 4.7, yields at once that the rate of convergence in question is not worse than the required one. What remains is to show that the above rate is optimal. This is accomplished in the proof below. Arguments applied in the case when the dimension of the space $\mathbb{H}^{n}$ is even are different from those when it is odd.

Theorem 4.9 We have

$$
P_{a}(x, y) \sim c|y|^{-2 n+2}, \quad|y| \rightarrow \infty
$$

Proof. Case $n=2 k$.

We will show that the formula (4.15) fails for $j=k$, i.e. that

$$
\int_{0}^{\infty} \kappa^{k} w_{\lambda}(v) d v \neq 0
$$


The formula (4.17) together with Lemmas 4.7 and 4.8 imply that

$$
\int_{0}^{\infty} w_{\lambda}(v) \Phi\left(\kappa /\left(\lambda^{2}+|y|^{2}\right)\right) d v \sim c|y|^{-2 k}=c|y|^{-n}
$$

$c \neq 0$, when $|y| \rightarrow \infty$. Taking into account the formula (4.10) we obtain the desired result. Observe that when $n=4$, the function $w_{\lambda}(v)=a^{-2} e^{-v}$ is positive so the formula (4.17) is apparent. As mentioned earlier, the exact convergence rate is easy to obtain directly from the formula (4.10). Thus, we assume throughout the proof that $k>2$.

Proof of the formula (4.17). Suppose that (4.17) is not true, i.e.

$$
\int_{0}^{\infty} \kappa^{k} w_{\lambda}(v) d v=0, \quad k>2
$$

Then, using Lemma 4.7 for $l=k+1$, it follows that there exists the limit

$$
\lim _{|y| \rightarrow \infty}|y|^{2 k+2} \int_{0}^{\infty} w_{\lambda}(v) \Phi\left(\frac{\kappa}{\left(\lambda^{2}+|y|^{2}\right)}\right) d v<\infty
$$

and, by (4.10),

$$
\lim _{|y| \rightarrow \infty}|y|^{2 n} P_{a}(x, y)=\frac{\Gamma\left(\frac{n}{2}-1\right)}{2 \pi^{n / 2}}<\infty .
$$

We will show that the existence of the limit (4.18) leads to a contradiction with the convergence of the Poisson kernels $P_{a}(x, y)$ to $P_{0}(x, y), a \rightarrow 0+$, established in Proposition 4.2.

By the homogeneity property of the kernel $P_{a}(x, y)$, proved in Corollary 2.3, we have

$$
P_{a^{2} / x}(a, a|y| / x)=(a / x)^{-n+1} P_{a}(x, y)
$$

so we also have

$$
\lim _{|y| \rightarrow \infty}|y|^{2 n} P_{a^{2} / x}(a, a y / x)<\infty
$$

We will prove that

$$
\limsup _{|y| \rightarrow \infty}|y|^{2 n} P_{a^{2} / x}(x, y)<\infty .
$$

Set $a_{1}=a^{2} / x$. As $0<a_{1}<a<x$, the semigroup property implies that

$$
|y|^{2 n} P_{a_{1}}(x, y)=\int_{\mathbb{R}^{n-1}} P_{a_{1}}(a, z)|y|^{2 n} P_{a}(x, z-y) d z .
$$

We divide the last integral into $\int_{2|z| \geqslant|y|}+\int_{2|z|<|y|}$ and estimate separately both integrals. 
By (4.19), we obtain that $\lim _{|y| \rightarrow \infty}|y|^{2 n} P_{a_{1}}(a, y)<\infty$. This is used in the estimate

$$
\begin{gathered}
\int_{2|z| \geqslant|y|} P_{a_{1}}(a, z)|y|^{2 n} P_{a}(x, z-y) d z \leqslant 2^{2 n} \int_{2|z| \geqslant|y|} P_{a_{1}}(a, z)|z|^{2 n} P_{a}(x, z-y) d z \\
\leqslant c 2^{2 n} \int_{2|z| \geqslant|y|} P_{a}(x, z-y) d z \leqslant c 2^{2 n}
\end{gathered}
$$

where the constant $c$ is common for all $|y|>M>0$. Next, observe that if $|y|>2|z|$ then $|y-z|>|y| / 2$, so that, using (4.18),

$$
\begin{gathered}
\int_{2|z|<|y|} P_{a_{1}}(a, z)|y|^{2 n} P_{a}(x, z-y) d z \leqslant 2^{2 n} \int_{2|z|<|y|} P_{a_{1}}(a, z)|z-y|^{2 n} P_{a}(x, z-y) d z \\
\leqslant c 2^{2 n} \int_{2|z|<|y|} P_{a_{1}}(a, z) d z \leqslant c 2^{2 n}
\end{gathered}
$$

and (4.20) is proved. Note that in order to prove it, a weaker hypothesis

$$
\limsup _{|y| \rightarrow \infty}|y|^{2 n} P_{a}(x, y)<\infty
$$

is sufficient. Consequently, denoting $q=a / x$ and iterating the last argument $j$ times we get

$$
\limsup _{|y| \rightarrow \infty}|y|^{2 n} P_{q^{j} a}(x, y)<\infty, \quad j \in \mathbb{N} .
$$

We denote $a_{j}=q^{j} a, \lambda_{j}=x-a_{j}, \kappa_{j}=\left(\lambda_{j}+a_{j} v\right)^{2}-\lambda_{j}^{2}$ and we denote $w_{\lambda_{j}}(v)$ the function appearing in the representation formula (3.7) for the kernel $P_{a_{j}}(x, y)$. The formula (4.21) implies that for all $j \in \mathbb{N}$

$$
\int_{0}^{\infty} \kappa_{j}^{n / 2} w_{\lambda_{j}}(v) d v=0
$$

(otherwise $|y|^{2 n-2} P_{a_{j}}(x, y)$ converges to a positive constant when $|y| \rightarrow \infty$, so $|y|^{2 n} P_{a_{j}}(x, y)$ diverges to $\left.+\infty\right)$.

Recall that

$$
\begin{aligned}
w_{\lambda_{j}}(v) & =w_{1, \lambda_{j}}(v)=\sum_{i=1}^{k_{n}}\left(\operatorname{Res}_{z_{i}} F_{\lambda_{j}}\right) e^{z_{i} v} \\
& =-\frac{\left(x / a_{j}\right)^{\frac{n-1}{2}}}{\lambda_{j} a_{j}} \sum_{i=1}^{k_{n}} \frac{z_{i} e^{x z_{i} / a_{j}} K_{\frac{n-1}{2}}\left(x z_{i} / a_{j}\right)}{e^{z_{i}} K_{\frac{n-3}{2}}\left(z_{i}\right)} e^{z_{i} v} .
\end{aligned}
$$


Writing

$$
\operatorname{Res}_{z_{i}} F_{\lambda_{j}}=-\frac{1}{a_{j}^{n / 2}} \frac{z_{i}^{1 / 2}}{\sqrt{2} \lambda_{j}} \frac{x^{n / 2-1}}{e^{z_{i}} K_{\frac{n-3}{2}}\left(z_{i}\right)}\left(2 \frac{x z_{i}}{a_{j}}\right)^{1 / 2} e^{x z_{i} / a_{j}} K_{\frac{n-1}{2}}\left(x z_{i} / a_{j}\right)
$$

and using the asymptotics $K_{\nu}(u)=\pi^{\frac{1}{2}}(2 u)^{-\frac{1}{2}} e^{-u}[1+E(u)], u \geqslant 1, E(u)=$ $O\left(u^{-1}\right)$ when $u \rightarrow \infty$, we get for $a_{j} \rightarrow 0+$

$$
a_{j}^{n / 2} \operatorname{Res}_{z_{i}} F_{\lambda_{j}}=-\left(\frac{\pi}{2}\right)^{1 / 2} \frac{z_{i}^{1 / 2}}{\left(x-a_{j}\right)} \frac{x^{n / 2-1}}{e^{z_{i}} K_{\frac{n-3}{2}}\left(z_{i}\right)}\left[1+E\left(\frac{x z_{i}}{a_{j}}\right)\right]
$$

and

$$
\tilde{w}(v):=\lim _{a_{j} \rightarrow 0} a_{j}^{n / 2} w_{\lambda}(v)=-\left(\frac{\pi}{2}\right)^{1 / 2} x^{n / 2-2} \sum_{i=1}^{k_{n}} \frac{z_{i}^{1 / 2} e^{z_{i} v}}{e^{z_{i}} K_{\frac{n-3}{2}}\left(z_{i}\right)} .
$$

Thus we get

$$
\lim _{j} \kappa_{j}^{n / 2} w_{\lambda_{j}}(v)=(2 x)^{n / 2} v^{n / 2} \tilde{w}(v) .
$$

By the Lebesgue dominated convergence theorem and by (4.22)

$$
\lim _{j} \int_{0}^{\infty} \kappa_{j}^{n / 2} w_{\lambda_{j}}(v) d v=(2 x)^{n / 2} \int_{0}^{\infty} v^{n / 2} \tilde{w}(v) d v=0 .
$$

Now, when $j \rightarrow \infty$ and $|y|$ is fixed, one has $\kappa_{j} \rightarrow 0$ and $u_{j}=\frac{\kappa_{j}}{\lambda_{j}^{2}+|y|^{2}} \rightarrow 0$. By (4.12) and by (4.23), we get

$$
\begin{aligned}
\lim _{j} \int_{0}^{\infty} & w_{\lambda_{j}}(v) \Phi\left(u_{j}\right) d v \\
& =\lim _{j} \int_{0}^{\infty} w_{\lambda_{j}}(v)\left[\Phi\left(u_{j}\right)-\sum_{2 \leqslant l \leqslant k-1}(-1)^{l} \frac{\left(\frac{n}{2}-1\right)_{l}}{l !} u_{j}^{l}\right] d v \\
& =(-1)^{n / 2} \frac{\left(\frac{n}{2}-1\right)_{n / 2}}{(n / 2) !}\left(\frac{2 x}{x^{2}+|y|^{2}}\right)^{n / 2} \int_{0}^{\infty} v^{n / 2} \tilde{w}(v) d v=0 .
\end{aligned}
$$

This implies that $\lim _{j} P_{a_{j}}(x, y)=0$ which is false because $\lim _{j} P_{a_{j}}(x, y)=$ $P_{0}(x, y) \neq 0$. The proof in the case $n=2 k$ is completed.

Case $n=2 k+1$. We denote $u=u(x, a, y, v)=\kappa /\left(\lambda^{2}+|y|^{2}\right)$. By Lemma 4.8 , for $n>3$

$$
\int_{0}^{\infty} w_{\lambda}(v) \Phi(u) d v=\int_{0}^{\infty} w_{\lambda}(v)\left[\Phi(u)-\sum_{2 \leqslant j \leqslant k}(-1)^{j} \frac{\left(\frac{n}{2}-1\right)_{j}}{j !} u^{j}\right] d v .
$$


This formula is also trivially true for $n=3$ (there is no sum on the righthand side). We want to show that

$$
\int_{0}^{\infty} w_{\lambda}(v) \Phi(u) d v \sim C|y|^{-n} \quad \text { when }|y| \rightarrow \infty
$$

for a positive constant $C$. We divide the last integral into the sum of three integrals

$$
\int_{0}^{\infty}=\int_{0}^{A}+\int_{A}^{\varepsilon\left(\lambda^{2}+|y|^{2}\right)^{\frac{1}{2}}}+\int_{\varepsilon\left(\lambda^{2}+|y|^{2}\right)^{\frac{1}{2}}}^{\infty},
$$

where $A>0$ is so big that

$$
(-1)^{\frac{n+1}{2}} w_{2, \lambda}(v)>\frac{c}{v^{n+1}}
$$

for a constant $c>0$; this is possible because, by Theorem $3.3,(-1)^{\frac{n+1}{2}} v^{n+1}$ $w_{2, \lambda}(v)$ converges when $v \rightarrow \infty$ to a positive constant. The value of $\epsilon$ will be chosen in the sequel. Recall that $k=\frac{n-1}{2}$.

We estimate the integral $\int_{0}^{A}$ using the bound (4.11) for $l=k+1$ and $\left|w_{\lambda}(v)\right| \leqslant c / v^{n+1}$. We obtain

$$
\begin{gathered}
\int_{0}^{A}\left|w_{\lambda}(v)\left[\Phi(u)-\sum_{2 \leqslant j \leqslant k}(-1)^{j} \frac{\left(\frac{n}{2}-1\right)_{j}}{j !} u^{j}\right]\right| d v \leqslant \frac{c}{\left(\lambda^{2}+|y|^{2}\right)^{k+1}} \int_{0}^{A} \frac{\kappa^{k+1}}{v^{n+1}} d v \\
\leqslant \frac{c_{1}}{|y|^{2(k+1)}} \int_{0}^{A} \frac{v^{2(k+1)}}{v^{n+1}} d v=\frac{c_{1} A}{|y|^{n+1}} .
\end{gathered}
$$

Next, observe that, again by (4.11) for $l=k+1$,

$$
\begin{aligned}
\int_{A}^{\infty}\left|w_{1, \lambda}(v)\left[\Phi(u)-\sum_{2 \leqslant j \leqslant k}(-1)^{j} \frac{\left(\frac{n}{2}-1\right)_{j}}{j !} u^{j}\right]\right| d v \\
\leqslant \frac{c}{\left(\lambda^{2}+|y|^{2}\right)^{k+1}} \int_{A}^{\infty}\left|w_{1, \lambda}(v)\right| \kappa^{k+1} d v \\
\leqslant \frac{c}{|y|^{n+1}} \int_{A}^{\infty}\left|w_{1, \lambda}(v)\right| v^{n+1} d v \leqslant \frac{c_{2}}{|y|^{n+1}}
\end{aligned}
$$

since the function $\left|w_{1, \lambda}(v)\right|$ decreases exponentially when $v \rightarrow \infty$. Thus, in order to show that

$$
\int_{0}^{\infty} w_{\lambda}(v) \Phi(u) d v \sim C|y|^{-n} \quad \text { when }|y| \rightarrow \infty
$$


it suffices to prove that

$$
\int_{A}^{\infty} w_{2, \lambda}(v)\left[\Phi(u)-\sum_{2 \leqslant j \leqslant k}(-1)^{j} \frac{\left(\frac{n}{2}-1\right)_{j}}{j !} u^{j}\right] d v \sim C|y|^{-n} .
$$

Note that by (4.13) with $l=k+1$ and by the fact that $(-1)^{\frac{n+1}{2}} w_{2, \lambda} \geqslant 0$, proved in Theorem 3.3, the integrand

$$
w_{2, \lambda}(v)\left[\Phi(u)-\sum_{2 \leqslant j \leqslant k}(-1)^{j} \frac{\left(\frac{n}{2}-1\right)_{j}}{j !} u^{j}\right]
$$

is non-negative.

Moreover, by (4.12), for $\epsilon>0$ sufficiently small and $v<\epsilon\left(\lambda^{2}+|y|^{2}\right)^{1 / 2}$, so that $v^{2} /\left(\lambda^{2}+|y|^{2}\right)<\epsilon^{2}, v /\left(\lambda^{2}+|y|^{2}\right) \leqslant v /\left(\lambda^{2}+|y|^{2}\right)^{1 / 2}<\epsilon$ and $u<b(x, a) \epsilon$, we have

$$
\left|\Phi(u)-\sum_{2 \leqslant j \leqslant k}(-1)^{j} \frac{\left(\frac{n}{2}-1\right)_{j}}{j !} u^{j}\right|>c u^{k+1},
$$

for a positive constant $c$. On the other hand, by (4.11), we have for all $u \geqslant 0$

$$
\left|\Phi(u)-\sum_{2 \leqslant j \leqslant k}(-1)^{j} \frac{\left(\frac{n}{2}-1\right)_{j}}{j !} u^{j}\right|<c^{\prime} u^{k+1} .
$$

We consider $|y|$ so big that $A<\epsilon\left(\lambda^{2}+|y|^{2}\right)^{1 / 2}$. The last two estimates imply

$$
\begin{aligned}
\frac{c}{\left(\lambda^{2}+|y|^{2}\right)^{k+1}} & \int_{A}^{\epsilon\left(\lambda^{2}+|y|^{2}\right)^{1 / 2}} \frac{\kappa^{k+1}}{v^{n+1}} d v \\
& \leqslant \int_{A}^{\epsilon\left(\lambda^{2}+|y|^{2}\right)^{1 / 2}} w_{2, \lambda}(v)\left[\Phi(u)-\sum_{2 \leqslant j \leqslant k}(-1)^{j} \frac{\left(\frac{n}{2}-1\right)_{j}}{j !} u^{j}\right] d v \\
& \leqslant \frac{c^{\prime}}{\left(\lambda^{2}+|y|^{2}\right)^{k+1}} \int_{0}^{\epsilon\left(\lambda^{2}+|y|^{2}\right)^{1 / 2}} \frac{\kappa^{k+1}}{v^{n+1}} d v,
\end{aligned}
$$

which, using $\kappa=a v(2 \lambda+a v)$, implies

$$
\begin{aligned}
\frac{c_{1}}{\left(\lambda^{2}+|y|^{2}\right)^{k+1}} \int_{A}^{\epsilon\left(\lambda^{2}+|y|^{2}\right)^{1 / 2}} d v & \int_{A}^{\epsilon\left(\lambda^{2}+|y|^{2}\right)^{1 / 2}} w_{2, \lambda}\left[\Phi(u)-\sum_{2 \leqslant j \leqslant k}(-1)^{j} \frac{\left(\frac{n}{2}-1\right)_{j}}{j !} u^{j}\right] d v \\
\leqslant & \frac{c_{2}}{\left(\lambda^{2}+|y|^{2}\right)^{k+1}} \int_{0}^{\epsilon\left(\lambda^{2}+|y|^{2}\right)^{1 / 2}} d v
\end{aligned}
$$




$$
\begin{aligned}
& \frac{c_{1} \epsilon}{\left(\lambda^{2}+|y|^{2}\right)^{k+(1 / 2)}}-\frac{c_{1} A}{\left(\lambda^{2}+|y|^{2}\right)^{k+1}} \\
& \quad \leqslant \int_{A}^{\epsilon\left(\lambda^{2}+|y|^{2}\right)^{1 / 2}} w_{2, \lambda}(v)\left[\Phi(u)-\sum_{2 \leqslant j \leqslant k}(-1)^{j} \frac{\left(\frac{n}{2}-1\right)_{j}}{j !} u^{j}\right] d v \\
& \quad \leqslant \frac{c_{2} \epsilon}{\left(\lambda^{2}+|y|^{2}\right)^{k+(1 / 2)}}
\end{aligned}
$$

and

$\frac{c_{3}}{|y|^{n}}-\frac{c_{4}}{|y|^{n+1}} \leqslant \int_{A}^{\epsilon\left(\lambda^{2}+|y|^{2}\right)^{1 / 2}} w_{2, \lambda}(v)\left[\Phi(u)-\sum_{2 \leqslant j \leqslant k}(-1)^{j} \frac{\left(\frac{n}{2}-1\right)_{j}}{j !} u^{j}\right] d v \leqslant \frac{c_{5}}{|y|^{n}}$.

Thus

$$
\int_{A}^{\epsilon\left(\lambda^{2}+|y|^{2}\right)^{1 / 2}} w_{2, \lambda}(v)\left[\Phi(u)-\sum_{2 \leqslant j \leqslant k}(-1)^{j} \frac{\left(\frac{n}{2}-1\right)_{j}}{j !} u^{j}\right] d v \sim C|y|^{-n}
$$

for $C>0,|y| \rightarrow \infty$.

In the last integral

$$
\int_{\epsilon\left(\lambda^{2}+|y|^{2}\right)^{1 / 2}}^{\infty} w_{2, \lambda}(v)\left[\Phi(u)-\sum_{2 \leqslant j \leqslant k}(-1)^{j} \frac{\left(\frac{n}{2}-1\right)_{j}}{j !} u^{j}\right] d v
$$

the variable $u$ is separated from 0 , so the expression

$$
\left|\Phi(u)-\sum_{2 \leqslant j \leqslant k}(-1)^{j} \frac{\left(\frac{n}{2}-1\right)_{j}}{j !} u^{j}\right|
$$

is estimated from above, up to a positive factor, by the highest level term $u^{k}$. Consequently

$$
\begin{aligned}
0 & <\int_{\epsilon\left(\lambda^{2}+|y|^{2}\right)^{1 / 2}}^{\infty} w_{2, \lambda}(v)\left[\Phi(u)-\sum_{2 \leqslant j \leqslant k}(-1)^{j} \frac{\left(\frac{n}{2}-1\right)_{j}}{j !} u^{j}\right] d v \\
& \leqslant \frac{c}{\left(\lambda^{2}+|y|^{2}\right)^{k}} \int_{\epsilon\left(\lambda^{2}+|y|^{2}\right)^{1 / 2}}^{\infty} \frac{\kappa^{k}}{v^{n+1}} d v \\
& \leqslant \frac{c^{\prime}}{\left(\lambda^{2}+|y|^{2}\right)^{k}} \int_{\epsilon\left(\lambda^{2}+|y|^{2}\right)^{1 / 2}}^{\infty} \frac{v^{n-1}}{v^{n+1}} d v=\frac{c^{\prime \prime}}{\left(\lambda^{2}+|y|^{2}\right)^{k}}\left(\lambda^{2}+|y|^{2}\right)^{-1 / 2} \sim \frac{C_{1}}{|y|^{n}}
\end{aligned}
$$


Since both integrals

$$
\begin{gathered}
\int_{A}^{\epsilon\left(\lambda^{2}+|y|^{2}\right)^{1 / 2}} w_{2, \lambda}(v)\left[\Phi(u)-\sum_{2 \leqslant j \leqslant k}(-1)^{j} \frac{\left(\frac{n}{2}-1\right)_{j}}{j !} u^{j}\right] d v \sim C|y|^{-n} \\
\int_{\epsilon\left(\lambda^{2}+|y|^{2}\right)^{1 / 2}}^{\infty} w_{2, \lambda}(v)\left[\Phi(u)-\sum_{2 \leqslant j \leqslant k}(-1)^{j} \frac{\left(\frac{n}{2}-1\right)_{j}}{j !} u^{j}\right] d v \leqslant C_{1}|y|^{-n}
\end{gathered}
$$

are positive, we conclude that

$$
\int_{A}^{\infty} w_{2, \lambda}(v)\left[\Phi(u)-\sum_{2 \leqslant j \leqslant k}(-1)^{j} \frac{\left(\frac{n}{2}-1\right)_{j}}{j !} u^{j}\right] d v \sim C|y|^{-n}
$$

and the proof in the case $n=2 k+1$ is finished.

We now deal with the remaining asymptotics of $P_{a}(x, y)$ near the boundary: $x \rightarrow a^{+},|y| \neq 0$.

Theorem 4.10 We have

$$
P_{a}(x, y) \sim c(x-a), \quad x \rightarrow a^{+}, \quad|y| \neq 0 .
$$

Proof. We recall the basic formula (4.9) for $P_{a}(x, y)$

$$
P_{a}(x, y)=\frac{\Gamma\left(\frac{n}{2}-1\right)}{2 \pi^{n / 2}} \frac{\lambda}{\left(\lambda^{2}+|y|^{2}\right)^{\frac{n}{2}-1}} \int_{0}^{\infty} w_{\lambda}(v) \Phi\left(\frac{\kappa}{\lambda^{2}+|y|^{2}}\right) d v .
$$

We apply here the notation and terminology introduced before the formulation of Theorem 4.9. In particular, we have for $0<\lambda \leqslant a$

$0 \leqslant \Phi\left(\frac{\kappa}{\lambda^{2}+|y|^{2}}\right) \leqslant\left(\frac{n}{2}-1\right) \frac{\kappa}{z}=\left(\frac{n}{2}-1\right) \frac{a v(2 \lambda+a v)}{\lambda^{2}+|y|^{2}} \leqslant\left(\frac{a}{v}\right)^{2} v(2+v)$.

We also have

$$
\lim _{x \rightarrow a+} \Phi\left(\frac{\kappa}{\lambda^{2}+|y|^{2}}\right)=\left(\frac{|y|^{2}}{|y|^{2}+(a v)^{2}}\right)^{\frac{n}{2}-1}-1+\left(\frac{n}{2}-1\right)\left(\frac{a v}{|y|}\right)^{2},
$$

and $\lim _{x \rightarrow a+} w_{\lambda}(v)=w(v)=w_{1}(v)+w_{2}(v)$.

Using properties of $w_{i, \lambda}$ stated in Theorem 3.3 one obtains

$$
\begin{aligned}
& \nu_{a}(y)=\lim _{x \rightarrow a+} \frac{P_{a}(x, y)}{\lambda} \\
& =\frac{\Gamma\left(\frac{n}{2}-1\right)}{2 \pi^{n / 2}|y|^{n-2}} \int_{0}^{\infty} w(v)\left[\left(\frac{|y|^{2}}{|y|^{2}+(a v)^{2}}\right)^{\frac{n}{2}-1}-1+\left(\frac{n}{2}-1\right)\left(\frac{a v}{|y|}\right)^{2}\right] d v .
\end{aligned}
$$


Using formulas (3.10) and (3.12) from the proof of Theorem 3.2 one computes

$$
1=\frac{a^{2}}{2} \int_{0}^{\infty} v^{2} w_{\lambda}(v) d v \rightarrow \frac{a^{2}}{2} \int_{0}^{\infty} v^{2} w(v) d v
$$

and

$$
\int_{0}^{\infty} w(v) d v=\lim _{x \rightarrow a+} \int_{0}^{\infty} w_{\lambda}(v) d v=\lim _{x \rightarrow a+} n(n-2)(x / a)^{\frac{n}{2}-1} \frac{1}{8 x a}=\frac{n(n-2)}{8 a^{2}} .
$$

We thus obtained

$$
\begin{aligned}
\nu_{a}(y) & =\lim _{x \rightarrow a+} \frac{P_{a}(x, y)}{\lambda} \\
& =\frac{\Gamma\left(\frac{n}{2}-1\right)}{2 \pi^{n / 2}}\left[\frac{n-2}{|y|^{n}}-\frac{n(n-2)}{8 a^{2}|y|^{n-2}}+\int_{0}^{\infty} \frac{w(v) d v}{\left(|y|^{2}+(a v)^{2}\right)^{\frac{n}{2}-1}}\right] .
\end{aligned}
$$

Observe now that when we multiply the right-hand side of the above equation by $|y|^{n-2}$ and let $|y| \rightarrow 0$ then the first term tends to infinity while the second one is constant and the third one converges to 0 :

$$
|y|^{n-2} \int_{0}^{\infty} \frac{w(v) d v}{\left(|y|^{2}+(a v)^{2}\right)^{\frac{n}{2}-1}}=\int_{0}^{\infty} \frac{w(v) d v}{\left(1+(a v /|y|)^{2}\right)^{\frac{n}{2}-1}} \rightarrow 0 .
$$

This shows that the density $\nu_{a}(y)$ does not vanish identically. We show that it is positive everywhere. This, however, requires some extra effort. We need the following formulas:

$$
\int_{0}^{\infty} e^{-|y|^{2} / 4 t} e^{-a^{2} v^{2} / 4 t} \frac{d t}{t^{\frac{n}{2}}}=\frac{2^{n-2} \Gamma\left(\frac{n}{2}-1\right)}{\left(a^{2} v^{2}+|y|^{2}\right)^{\frac{n}{2}-1}}
$$

and

$$
\int_{0}^{\infty} e^{-|y|^{2} / 4 t} \frac{d t}{t^{\frac{n}{2}}}=\frac{2^{n-2} \Gamma\left(\frac{n}{2}-1\right)}{|y|^{n-2}}, \quad \int_{0}^{\infty} e^{-|y|^{2} / 4 t} \frac{d t}{t^{1+\frac{n}{2}}}=\frac{2^{n} \Gamma\left(\frac{n}{2}\right)}{|y|^{n}} .
$$

The above formulas as well as the form of the function $\nu_{a}(y)$ enable us to transform (4.24) into the following expression:

$$
\begin{aligned}
\nu_{a}(y) & =\frac{2}{(4 \pi)^{\frac{n}{2}}} \int_{0}^{\infty} e^{-\frac{|y|^{2}}{4 t}} \int_{0}^{\infty}\left[e^{-a^{2} v^{2} / 4 t}-1+\frac{a^{2} v^{2}}{4 t}\right] w(v) d v \frac{d t}{t^{n / 2}} \\
& =\int_{0}^{\infty} \frac{1}{(4 \pi t)^{\frac{n-1}{2}}} e^{-\frac{|y|^{2}}{4 t}}\left(\frac{1}{\sqrt{\pi t}} \int_{0}^{\infty}\left[e^{-a^{2} v^{2} / 4 t}-1+\frac{a^{2} v^{2}}{4 t}\right] w(v) d v\right) d t \\
& =\int_{0}^{\infty} \frac{1}{(4 \pi t)^{\frac{n-1}{2}}} e^{-\frac{|y|^{2}}{4 t}} m_{a}(t) d t
\end{aligned}
$$


Here

$$
m_{a}(t)=\frac{1}{\sqrt{\pi t}} \int_{0}^{\infty}\left[e^{-a^{2} v^{2} / 4 t}-1+\frac{a^{2} v^{2}}{4 t}\right] w(v) d v
$$

is nonnegative (see Corollary 4.12 below). Observe that $\sqrt{t} m_{a}(t)$ has the following holomorphic extension for the halfplane $\Re(z)>0$ :

$$
\frac{1}{\sqrt{\pi}} \int_{0}^{\infty}\left[e^{-a^{2} v^{2} / 4 z}-1+a^{2} v^{2} / 4 z\right] w(v) d v
$$

It follows that $m_{a}(t)$ may only have isolated zeros, except possibly at 0 . In particular, $m_{a}(t)>0$ almost everywhere. So, $\nu_{a}(y)>0$ for all $a>0$ and $|y|>0$, which completes the proof.

The following result, indispensable to complete the proof of Theorem 4.10, is taken from the paper [4].

Lemma 4.11 Let

$$
q_{\frac{n-1}{2}}(t)=\frac{\lambda}{\sqrt{\pi t}} \int_{0}^{\infty}\left[e^{-\kappa / 4 t}-1+\kappa / 4 t\right] w_{\lambda}(v) d v .
$$

The function $q_{\frac{n-1}{2}}(t)$ is a probabilistic density.

Remark. The function $q_{\frac{n-1}{2}}(t)$ is the density of the distribution of the functional $A\left(\tau_{a}\right)$, where

$$
A(t)=x^{2} \int_{0}^{t} \exp 2(B(s)-(n-2) s) d s,
$$

$B(t)$ is the standard Brownian motion (with variance $2 t$ ) and $\tau_{a}$ is the first hitting time of the level $a<x$ by the geometric Brownian motion $X(t)=$ $x \exp (B(t)-(n-2) t)$. The formula $(4.25)$ is one of the main results of the paper [4]. It turns out (and is well known) that the Poisson kernel is the density of distribution of the process $Y_{A\left(\tau_{a}\right)}$, where $Y$ is the standard $(n-1)$-dimensional Brownian motion, independent from $B$. The function $m_{a}(t)$ is obtained from $q_{\frac{n-1}{2}}(t)$ via limiting procedure in the same way as the function $\nu_{a}(y)$ and we state this fact here for the sake of convenience as

Corollary 4.12 The following holds

$$
m_{a}(t)=\lim _{x \rightarrow a^{+}} \frac{q_{\frac{n-1}{2}}(t)}{\lambda}
$$

The information that $m_{a}(t) \geqslant 0$ is essential for completing of the proof of the last theorem. 


\section{Acknowledgements}

The authors are deeply grateful to the referee for critical remarks and comments which enabled them to improve the presentation of the paper. The authors would like also to thank K. Bogdan, T. Kulczycki, M. Ryznar and T. Żak for stimulating conversations on the subject. Moreover, the first and the third named authors acknowledge the hospitality of Université d'Angers where a substantial part of the work was done. The third named author wishes to express his gratitude to the MAPMO laboratory at Orléans for giving the opportunity to work further on this subject during his post-doctoral fellowship.

\section{References}

[1] Baldi, P., Casadio Tarabusi, E. and Figà-Talamanca, A.: Stable laws arising from hitting distributions of processes on homogeneous trees and the hyperbolic half-plane. Pacific J. Math. 197 (2001), 257-273.

[2] Baldi, P., Casadio Tarabusi, E., Figà-Talamanca, A. and Yor, M.: Non-symmetric hitting distributions on the hyperbolic half-plane and subordinated perpetuities. Rev. Mat. Iberoamericana 17 (2001), 587-605.

[3] Bougerol, P. And Jeulin, T.: Brownian bridge on hyperbolic spaces and on homogeneous trees. Probab. Theory Related Fields 115 (1999), 95-120.

[4] Byczkowski, T. and Ryznar, M.: Hitting distributions of geometric Brownian motion. Studia Math. 173 (2006), 19-38.

[5] Chung, K. L. and Zhao, Z.: From Brownian motion to Schrödinger's equation. Fundamental Principles of Mathematical Sciences 312. SpringerVerlag, Berlin, 1995.

[6] Dufresne, D.: The distribution of a perpetuity, with application to risk theory and pension funding. Scand. Actuar. J. (1990), 39-79.

[7] Erdélyi, A., Magnus, W., Oberhettinger, F. and Tricomi, F. G.: Tables of integral transforms. Vol. I and II. McGraw-Hill Book Company, Inc., New York-Toronto-London, 1954.

[8] Erdélyi, A., Magnus, W., Oberhettinger, F. and Tricomi, F. G.: Higher Transcendental Functions. Vol. II. McGraw-Hill Book Company, New York-Toronto-London, 1953.

[9] Folland, G. B.: Fourier analysis and its applications. The Wadsworth \& Brooks/Cole Mathematics Series. Wadsworth \& Brooks/Cole Advanced Books \& Software, Pacific Grove, CA, 1992.

[10] Gradstein, I. S. And Ryzhik, I. M.: Table of integrals, series and products. Sixth edition, Academic Press, London 2000.

[11] Guivarc'H, Y., Ji, L. And Taylor, J. C.: Compactifications of symmetric spaces. Progress in Mathematics 156. Birkhäuser, Boston, 1998. 
[12] Helgason, S.: Groups and geometric analysis. Integral geometry, invariant differential operators, and spherical functions. Mathematical Surveys and Monographs 83. American Mathematical Society, Providence, RI, 2000.

[13] Yor, M.: Sur certaines fonctionnelles du mouvement Brownien réel. J. Appl. Probab. 29 (1992), 202-208.

[14] Yor, M.: On some exponential functionals of Brownian Motion. Adv. in Appl. Probab. 24 (1992), 509-531.

[15] Yor, M. (ed.): Exponential functionals and principal values related to Brownian motion. Biblioteca de la Revista Matemática Iberoamericana, Madrid, 1997.

Recibido: 17 de noviembre de 2004

Revisado: 20 de junio de 2005

Tomasz Byczkowski

Institute of Mathematics

Wrocław University of Technology

Wyb. Wyspiańskiego 27

50-370 Wrocław, Poland

Tomasz.Byczkowski@pwr . wroc.pl

Piotr Graczyk

Département de Mathématiques

Université d'Angers

2 bd. Lavoisier

49045 Angers cedex 1, France

graczyk@univ-angers.fr

Andrzej Stós

Institute of Mathematics

Wrocław University of Technology

and

Laboratoire de Mathématique, UMR 6620

Université Blaise Pascal

Campus des Cézeaux, 24 av. des Landais 63177 Aubière cedex, France

stos@math . univ-bpclermont . fr

Research partially supported by KBN grant 2 P03A 04122 and RTN Harmonic Analysis and Related Problems contract HPRN-CT-2001-00273-HARP. 